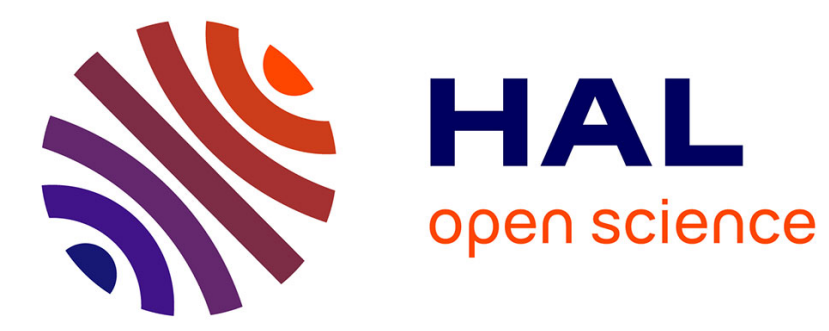

\title{
Mg2+-dependent conformational equilibria in CorA: an integrated view on transport regulation
}

Nicolai Tidemand Johansen, Marta Bonaccorsi, Tone Bengtsen, Andreas

Haahr Larsen, Frederik Grønbæk Tidemand, Martin Cramer Pedersen, Pie

Huda, Jens Berndtsson, Tamim Darwish, Nageshwar Rao Yepuri, et al.

\section{To cite this version:}

Nicolai Tidemand Johansen, Marta Bonaccorsi, Tone Bengtsen, Andreas Haahr Larsen, Frederik Grønbæk Tidemand, et al.. Mg2+-dependent conformational equilibria in CorA: an integrated view on transport regulation. 2021. hal-03455581

\section{HAL Id: hal-03455581 \\ https://hal.science/hal-03455581}

Preprint submitted on 29 Nov 2021

HAL is a multi-disciplinary open access archive for the deposit and dissemination of scientific research documents, whether they are published or not. The documents may come from teaching and research institutions in France or abroad, or from public or private research centers.
L'archive ouverte pluridisciplinaire HAL, est destinée au dépôt et à la diffusion de documents scientifiques de niveau recherche, publiés ou non, émanant des établissements d'enseignement et de recherche français ou étrangers, des laboratoires publics ou privés. 
$1 \mathrm{Mg}^{2+}$-dependent conformational equilibria in CorA: an

2 integrated view on transport regulation

3 Nicolai Tidemand Johansen ${ }^{*, 1}$, Marta Bonaccorsi ${ }^{*, 2}$, Tone Bengtsen ${ }^{*, 3,4}$, Andreas Haahr

4 Larsen ${ }^{1,4}$, Frederik Grønbæk Tidemand ${ }^{1}$, Martin Cramer Pedersen ${ }^{1}$, Pie Huda ${ }^{5}$, Jens

5 Berndtsson $^{6}$, Tamim Darwish ${ }^{7}$, Nageshwar Rao Yepuri ${ }^{7}$, Anne Martel ${ }^{8}$, Thomas Günther

6 Pomorski $^{9,10}$, Andrea Bertarello ${ }^{2}$, Mark Sansom ${ }^{4}$, Mikaela Rapp ${ }^{6}$, Ramon Crehuet ${ }^{3}$, Tobias

7 Schubeis ${ }^{\#, 2}$, Kresten Lindorff-Larsen ${ }^{\#, 3}$, Guido Pintacuda ${ }^{\#, 2}$, and Lise Arleth ${ }^{\#, 1}$

$9 \quad$ *Equally contributing authors

10 "Corresponding author

11 Emails: tobias.schubeis@ens-lyon.fr, lindorff@bio.ku.dk, guido.pintacuda@ens-lyon.fr,

12 arleth@nbi.ku.dk

1 Condensed Matter Physics, Niels Bohr Institute, University of Copenhagen, Copenhagen,

15 Denmark

162 Centre de RMN à Très hauts Champs de Lyon (UMR 5280, CNRS / Ecole Normale

Supérieure de Lyon / Université Claude Bernard Lyon 1), University of Lyon, Villeurbanne, France

3 Structural Biology and NMR Laboratory and Linderstrøm-Lang Centre for Protein Science,

5 Australian Institute for Bioengineering and Nanotechnology, The University of Queensland, Brisbane, Australia

6 Department of Biochemistry and Biophysics, Center for Biomembrane Research, Stockholm University, Stockholm, Sweden Lucas Heights, Australia

288 Institut Laue-Langevin, Grenoble, France

9 Section for Transport Biology, Department of Plant and Environmental Sciences, 


\section{Abstract}

The CorA family of proteins regulates the homeostasis of divalent metal ions in many bacteria, archaea, and eukaryotic mitochondria, making it an important target in the investigation of the mechanisms of transport and its functional regulation. Although numerous structures of open and closed channels are now available for the CorA family, the mechanism of the transport regulation remains elusive. Here, we investigated the conformational distribution and associated dynamic behaviour of the pentameric $\mathrm{Mg}^{2+}$ channel CorA at room temperature using small-angle neutron scattering (SANS) in combination with molecular dynamics (MD) simulations and solid-state nuclear magnetic resonance spectroscopy (NMR). We find that neither the $\mathrm{Mg}^{2+}$-bound closed structure nor the $\mathrm{Mg}^{2+}$-free open forms are sufficient to explain the average conformation of CorA. Our data support the presence of conformational equilibria between multiple states, and we further find a variation in the behaviour of the backbone dynamics with and without $\mathrm{Mg}^{2+}$. We propose that CorA must be in a dynamic equilibrium between different non-conducting states, both symmetric and asymmetric, regardless of bound $\mathrm{Mg}^{2+}$ but that conducting states become more populated in $\mathrm{Mg}^{2+}$-free conditions. These properties are regulated by backbone dynamics and are key to understanding the functional regulation of CorA.

\section{Introduction}

Magnesium is the most abundant divalent cation $\left(\mathrm{Mg}^{2+}\right)$ inside the cell, where it is mainly associated with the biological energy source adenosine triphosphate and other negatively charged molecules ${ }^{1} . \mathrm{Mg}^{2+}$ serves several biological functions, e.g. as co-factor for enzymes ${ }^{1}$, and $\mathrm{Mg}^{2+}$ deficiency is linked to severe diseases including cardiac syndromes, muscular dysfunction and bone wasting ${ }^{2-4}$. CorA is the main ion channel for $\mathrm{Mg}^{2+}$-import in most bacteria and archea $^{5}$. Despite little sequence conservation, CorA shares two membrane spanning helices and a conserved GMN motif with eukaryotic homologs, including Mrs2 that is responsible for $\mathrm{Mg}^{2+}$-import to the mitochondrial lumen and is essential for cell survival ${ }^{6,7}$.

Several structures determined by X-ray crystallography are available for Thermotoga maritima CorA (TmCorA $)^{8-12}$. All wild-type proteins have been crystalized as nearly symmetric pentamers in the presence of divalent metal ions and all represent a non-conducting state of the channel with a narrow and hydrophobic pore. Figure 1A shows a representative structure, which is characterized by a transmembrane domain (TMD) connected to the intracellular domain (ICD) by a long stalk helix. The periplasmic entrance to the pore contains the 
conserved GMN motif that presumably binds to $\mathrm{Mg}^{2+}$ via its first hydration shell and thereby acts as a selectivity filter ${ }^{11,13,14}$. The ICD contains ten inter-protomer binding sites for $\mathrm{Mg}^{2+}$ (two per protomer, denoted M1 and M2) involved in regulating the channel ${ }^{11,15,16}$. The open state(s) of CorA have so far not been crystallized, but several biochemical and structural studies ${ }^{15,16}$ as well as molecular dynamics simulations ${ }^{12}$ have pinpointed the determining residues involved in gating and suggested open models. One model suggests pore dilation upon loss of $\mathrm{Mg}^{2+}$ at the M1 (and M2) sites due to a concerted iris-like movement ${ }^{16,17}$, while another suggests a hydrophobic-to-polar transition of the pore upon concerted rotation of the stalk helices ${ }^{18,19}$.

Recently, cryo-EM structures were obtained both in the presence and absence of $\mathrm{Mg}^{2+20}$. The $\mathrm{Mg}^{2+}$ bound structure at $\sim 3.8 \AA$ resolution was symmetric and closed, in line with crystal structures, whereas two $\mathrm{Mg}^{2+}$-free structures at $\sim 7.1 \AA$ were symmetry broken and with dilated pores. Figure 1B shows an intracellular view of the symmetric and asymmetric states, highlighting the symmetry break upon removing $\mathrm{Mg}^{2+}$. From these observations, the proposed model involves a sequential destabilisation of CorA upon $\mathrm{Mg}^{2+}$ removal, leading to a highly dynamic protein with shuffling protomers in the ICD, increasing the likelihood of pore dilation and wetting events ${ }^{20,21}$. Recent coarse grained MD simulations revealed the residue level details of how a complex interaction network involving asymmetric movements of ICD monomers ultimately leads to a conducting state upon removal of $\mathrm{Mg}^{2+22}$. High-speed atomic force microscopy (HS-AFM) data on densely packed CorA in lipid bilayers supported this model, but at the same time provided more insight to the dynamic interconversion of different states, including a fourth population of highly asymmetric CorA, not resolved by cryo-EM ${ }^{23}$. Interestingly, this population accounted for most observed conformations at low $\mathrm{Mg}^{2+}$ concentrations, supporting that CorA is a dynamic protein with a relatively flat energy landscape and, potentially, multiple open states. However, CorA mutants with mutated regulatory $\mathrm{M} 1$ sites were still able to crystallize in the (symmetric) closed state ${ }^{19}$, suggesting that inter-protomer binding of $\mathrm{Mg}^{2+}$ is not required for closing the channel. Overall, the cryoEM and AFM experiments hint towards a highly dynamic ensemble of primarily asymmetric states at low $\mathrm{Mg}^{2+}$ concentrations, while the successful crystallisation of $\mathrm{M} 1$ site mutants suggests that the closed state is significantly present at these conditions.

In this study, we investigated CorA using two room-temperature methods, namely small-angle neutron scattering (SANS), sensitive to large amplitude conformational changes and magicangle spinning solid-state NMR (MAS NMR), sensitive to structure and dynamics with atomic resolution $^{24,25}$. For both methods, we employ custom developed state-of-the-art methodology, 
i.e. size-exclusion chromatography (SEC) coupled to $\mathrm{SANS}^{26,27}$ and match-out deuterated carrier systems for SANS ${ }^{28,29}$ (so-called stealth carrier systems), and >100 kHz MAS NMR in lipid bilayers ${ }^{30,31}$. Based on these data in conjunction with molecular simulations and modelling, we propose a model in which CorA is in a dynamic equilibrium between symmetric and asymmetric states, independent of bound $\mathrm{Mg}^{2+}$, but where an ensemble of conducting states is energetically more favourable for $\mathrm{Mg}^{2+}$-free CorA due to increased conformational dynamics resulting from the released electrostatic constraint.

111

112

113

114

115

116
A

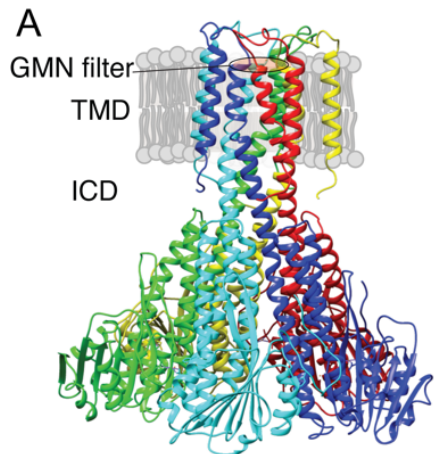

B
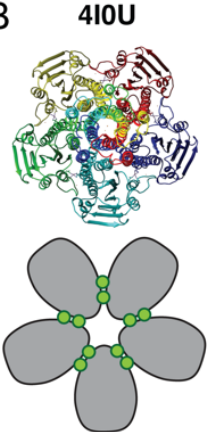

3JCG
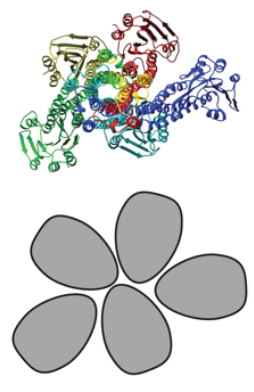

Figure $1 X$-ray and cryo-EM structures of CorA. A: Side view of symmetric CorA (PDB ID: $4 I 0 U$ ) in presence of $\mathrm{Mg}^{2+}$ ("closed form"). B: Top view of the same symmetric state of CorA (PDB ID: 4IOU) side-by-side to one of the asymmetric states observed in the absence of $\mathrm{Mg}^{2+}$ ("open form") (PDB ID: 3JCG). A schematic representation of the two forms is shown below their structures, with each monomer shown in gray and $\mathrm{Mg}^{2+}$ ions represented as green circles.

\section{Results}

\section{CorA is structurally similar in presence and absence of $\mathbf{M g}^{2+}$}

119 The published cryo-EM structures of CorA in absence of $\mathrm{Mg}^{2+}$ (Figure 1B, 3JCG) reveal large structural rearrangements compared to the nearly symmetric, non-conductive state obtained from crystallography (Figure 1B, 4IOU). SANS curves calculated from these two structural states of CorA reveal a significant change in the scattering curve in the region $q=0.08 \AA^{-1}$ $0.15 \AA^{-1}$ (Figure $2 A$ and Figure $2 B$, right panels), i.e. on a length scale that is well-covered in a standard SANS experiment. To match the cryo-EM conditions, we performed SANS measurements in n-dodecyl-B-D-maltoside (DDM) detergent micelles and 2-Oleoyl-1palmitoyl-sn-glycero-3-phosphocholine (POPC) lipid nanodiscs. We used selectively deuterated versions of both carrier types that were homogenously matched-out and hence invisible at $100 \% \mathrm{D}_{2} \mathrm{O}$; i.e. stealth DDM (sDDM) and stealth nanodiscs (sND, Figure 2-figure supplement 1). Strikingly, the measured SANS curves are pair-wise indistinguishable in the absence of $\mathrm{Mg}^{2+}$ (1 mM EDTA) and in the presence of $40 \mathrm{mM} \mathrm{Mg}^{2+}$ for the sDDM (Figure 2A) and SND (Figure 2B) samples, respectively, indicating no significant difference in the average conformations of the $\mathrm{Mg}^{2+}$-free and bound states of CorA. This observation contrasts with the 
recently proposed large-scale structural rearrangements reported from cryo-EM and highspeed AFM data.

We note that the SANS data obtained on the SND samples (Figure 2B) have a slight excess scattering contribution at low- $q$ compared to the sDDM samples (Figure 2A), which can be attributed to the presence of a few $E$. coli endogenous lipids in the SND samples. However, the SANS data from SDDM and sND samples are indistinguishable in the $q$-region expected to reveal differences from symmetric and asymmetric states (Figure 2-figure supplement 2), which confirms that CorA exhibits the same behaviour in a POPC lipid environment and in DDM detergent carriers. The rightmost panels of Figure 2A and Figure 2B show enhanced views on this region for the SANS data compared to the SANS curves calculated from the PDB structures. Interestingly, neither of the curves calculated from the PBD structures match the measured SANS data, suggesting that the solution structure of CorA cannot be described by any of these single structures, regardless of whether or not CorA is in the presence of $\mathrm{Mg}^{2+}$.

While SANS data provided information on the overall molecular shape of CorA in the two preparations, we used MAS NMR to obtain insight into structural changes at the residue-level length-scale. MAS NMR data were recorded on uniformly ${ }^{13} \mathrm{C},{ }^{15} \mathrm{~N}$-labelled CorA, reconstituted in 1,2-dimyristoyl-sn-glycero-3-phosphocholine (DMPC) lipid bilayers, in the presence or absence of $\mathrm{Mg}^{2+}$. Backbone resonance assignment was obtained at high $\mathrm{Mg}^{2+}$ concentration acquiring a set of three-dimensional experiments relying on ${ }^{1} \mathrm{H}^{\mathrm{N}}$ and ${ }^{1} \mathrm{Ha}$ detection with 100 $\mathrm{kHz}$ MAS. We were able to annotate $\sim 100$ peaks to residues spread throughout the structure of CorA. Notably, the assignment of CorA with and without $\mathrm{Mg}^{2+}$ is clustered in the globular region in the ICD and in the $T M D$, including the important periplasmic loop, whereas only sparse assignments were established in the long portion (243-289) of the stalk helix connecting the two regions. The determination of random coil chemical shift deviation (CSD) values confirmed that the secondary structure is in good agreement with the one obtained by X-ray crystallography and Cryo-EM.

$2 \mathrm{D}{ }^{1} \mathrm{H}-{ }^{15} \mathrm{~N}$ dipolar correlation spectra represent direct structural "fingerprints" of CorA in the two preparations. Despite the high signal overlap associated to the high molecular weight of CorA, fast MAS rates and the ultra-high magnetic field guarantee high sensitivity and feature numerous signals with a resolution sufficient to track subtle structural changes. Once again, against our expectations, we remarked that the spectra with and without $\mathrm{Mg}^{2+}$ showed very little difference, with the positions of the resolved peaks differing less than $0.1 \mathrm{ppm}$ in the two forms and without peak splitting or broadening that would indicate distinct conformations (Figure 2C). 
170 Two parallel strategies were pursued to extend the analysis to the more crowded regions.

171 First, we acquired three-dimensional (3D) experiments which correlate the amide proton and

172 nitrogen with the $\mathrm{C}_{\alpha}$-carbon within each residue and thus include an additional ${ }^{13} \mathrm{C}$ chemical 173 shift dimension (Figure 2D). The resulting 3D spectra confirmed negligible chemical shift variations over more than $\sim 90$ sites across the TMD and ICD. In the $\mathrm{Mg}^{2+}$-free sample, however, a notable decrease in signal intensity was observed for most residues, resulting in the complete disappearance of two thirds of the peaks from the TMD (vide infra).

177 Secondly, we used amino acid-specific isotopic enrichment to select the NMR signals associated to the amide groups of the alanine residues. Each CorA protomer contains eight alanine residues, distributed with four in the TMD and four in the ICD, which were all visible and assigned in the corresponding ${ }^{1} \mathrm{H}-{ }^{15} \mathrm{~N}$ dipolar correlation spectra of two preparations with and without magnesium (Figure 2E). Also, in this case, the spectra are superimposable and show no evidence of peak splitting.

In conclusion, and in line with SANS, the NMR data show that the predominant structure of CorA in lipid bilayers is unaltered by the removal of $\mathrm{Mg}^{2+}$.

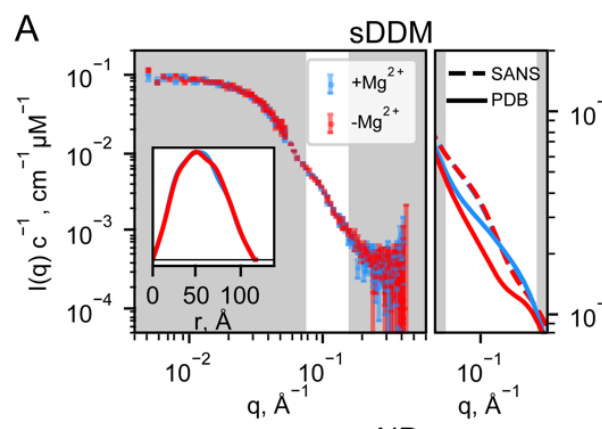

\section{C}
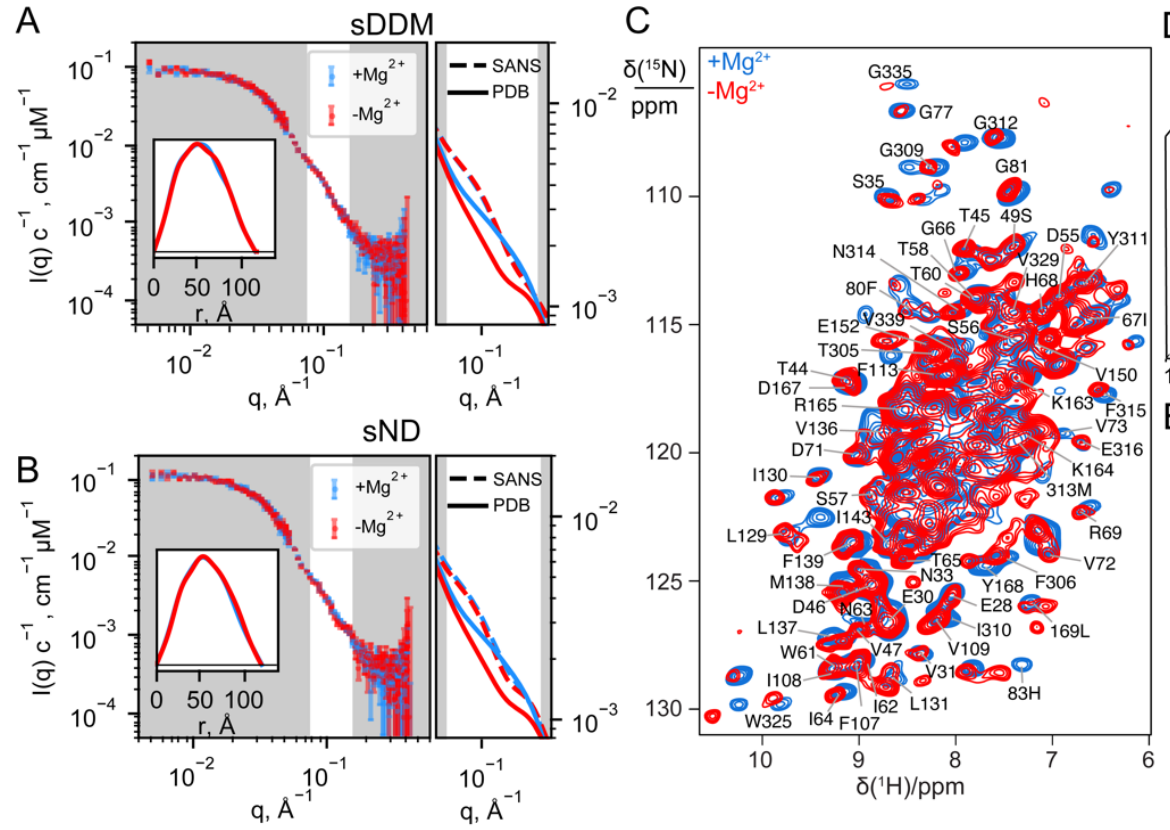

D

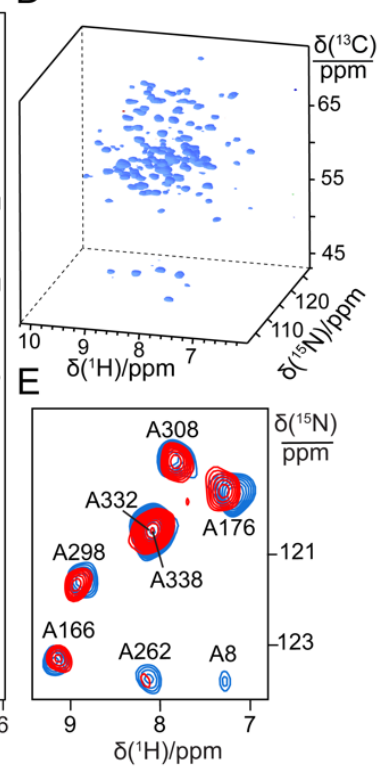

Figure 2 Experimental data on CorA in presence (blue) and absence (red) of $\mathrm{Mg}^{2+} . \mathrm{A}+\mathrm{B}$ : Experimental SANS data of CorA embedded in stealth DDM micelles (sDDM) and stealth nanodiscs ( $S N D)$, respectively, with $p(r)$-distributions calculated on BayesApp ${ }^{32}$ in the inset. The rightmost plots show zoomed comparisons of the $p(r)$-fits of experimental data (SANS, dashed lines) with the SANS curves calculated on the X-ray (4IOU) and cryo-EM (3JCG) structures (PDB, full lines). Complete fits based on the PDB structures are shown in Figure 4A. C: $2 D^{1} \mathrm{H}^{-15} \mathrm{~N}$ dipolar correlation spectra by MAS NMR of CorA embedded in hydrated DMPC bilayers recorded at $1 \mathrm{GHz}{ }^{1} \mathrm{H}$ Larmor frequency and $107 \mathrm{kHz}$ MAS. D: Cube representation of a $3 D{ }^{1} \mathrm{H}^{15} \mathrm{~N}-{ }^{13} \mathrm{C} \alpha$ spectrum. E: $2 D{ }^{1} \mathrm{H}-{ }^{15} \mathrm{~N}$ dipolar correlation spectra obtained for ${ }^{15} \mathrm{~N}$-Alanine labeled CorA recorded at $1 \mathrm{GHz}{ }^{1} \mathrm{H}$ Larmor frequency and $60 \mathrm{kHz}$ MAS. In $C$ and $E$, site-specific assignments are annotated for resolved resonances. 
Figure 2-figure supplement 1 Validation of nanodisc match-out deuteration. A: Background subtracted SANS data measured on sNDs in different percentage of $D_{2} \mathrm{O}$. B: Comparison of unsubtracted SANS data and corresponding background measurement in $100 \% \mathrm{D}_{2} \mathrm{O}$. C: Plot of contrast vs $D_{2} \mathrm{O}$ to verify match-point of sample (fitted to $99.9 \% \mathrm{D}_{2} \mathrm{O}$ by linear regression). I(0) values were determined using Guinier fits to the low-q data (grey points) as highlighted in the insert. The Guinier fit to the SANS data at $100 \% D_{2} \mathrm{O}$ has not been shown due to the large errorbars, and the fit was also not included for fitting the contrast vs $D_{2} O$. D: SAXS data on $s N D$ collected in $\mathrm{H}_{2} \mathrm{O}$ buffer together with $\mathrm{p}(r)$ distribution (insert). A nanodisc mode/ ${ }^{33}$ was fitted to the data using WilltFit ${ }^{34}$, producing a great fit, as evaluated by the residual plot on the bottom.

Figure 2-figure supplement 2 Comparison of SANS data in sDDM and SND. Same data as shown in Figure 1D-E, but presented here to compare data collected in sDDM vs sND. Despite the slightly higher forward scattering in the sND data, the SANS data from the two different systems overlap in the region of interest, as highlighted. Given that CorA had spent less time and steps in presence of DDM prior to reconstitution in sNDs, the excess signal likely stems from a small number of tightly bound $E$. coli lipids that do not match out in $D_{2} O$. By comparing the extrapolated values of I(O) between the sDDM and sND samples, we estimated the number to be on the order of 10 lipids, i.e. two lipids per protomer. Although the presence of visible lipids would complicate further detailed analysis, the striking identicality of the measured curves with and without $\mathrm{Mg}^{2+}$ in sNDs supports no large structural rearrangements.

\section{CorA is active and preserves its tertiary structure in $\mathrm{D}_{\mathbf{2}} \mathrm{O}$}

221 Since $\mathrm{Mg}^{2+}$ hydration plays an important role in CorA selectivity, and $\mathrm{D}_{2} \mathrm{O}$ and $\mathrm{H}_{2} \mathrm{O}$ have slightly different physicochemical properties ${ }^{35}$, we speculated whether the identical SANS curves with and without $\mathrm{Mg}^{2+}$ were due to CorA losing its activity in the SANS condition, i.e. at $100 \% \mathrm{D}_{2} \mathrm{O}$. To test this, we measured the activity of CorA in POPC liposomes under the SANS conditions by a fluorometric assay (Figure $3 \mathrm{~A}$ ). This analysis shows that CorA is clearly active in $\mathrm{D}_{2} \mathrm{O}$. The transport rate estimated from the initial linear part of the trace is lower by less than a factor of two compared to $\mathrm{H}_{2} \mathrm{O}$. A slightly reduced rate in $\mathrm{D}_{2} \mathrm{O}$ has been reported for other membrane proteins ${ }^{36}$ and is explainable by slightly altered properties of the two solvents ${ }^{35,37}$. We could also inhibit CorA activity in $\mathrm{D}_{2} \mathrm{O}$ with $\mathrm{Co}[\mathrm{NH} 3]_{6}{ }^{3+}$ (Figure $3 \mathrm{~A}$, green), supporting that the protein is indeed functional under the SANS conditions.

We further carried out negative stain EM on samples of CorA in DDM and $\mathrm{D}_{2} \mathrm{O}$ with or without $\mathrm{Mg}^{2+}$. The refined $2 \mathrm{D}$ classes clearly show that the pentameric architecture of CorA is preserved in $\mathrm{D}_{2} \mathrm{O}$ in both conditions (Figure 3B). Furthermore, several 2D classes appear to exhibit approximate fivefold symmetry, both in the presence and absence of $\mathrm{Mg}^{2+}$. To avoid bias, we refined 3D models without imposing symmetry (Figure 3C). In both conditions, these low-resolution $3 \mathrm{D}$ models $(\approx 15 \AA)$ are reminiscent of the overall expected architecture of CorA (Figure 1), but notably show some degree of asymmetry. In conclusion, CorA show little to no perturbation from measurements in $\mathrm{D}_{2} \mathrm{O}$, rendering the SANS data sets viable for modelling of the solution structure of CorA. 


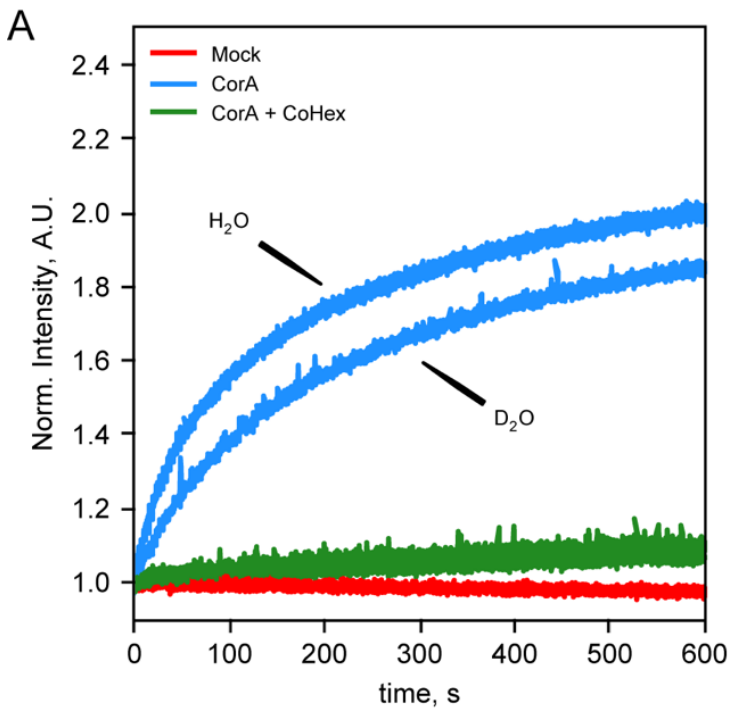

$\mathrm{B}+\mathrm{Mg}^{2+}$ $-\mathrm{Mg}^{2+}$
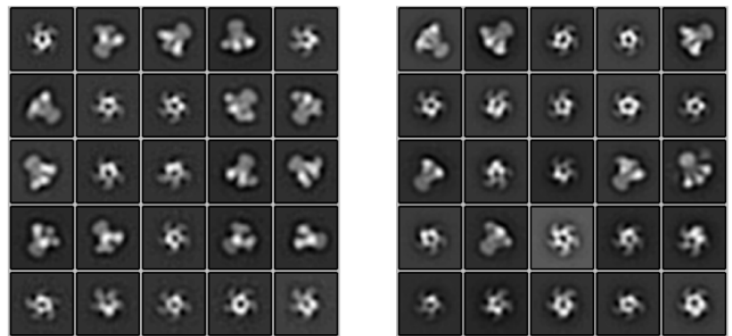

C
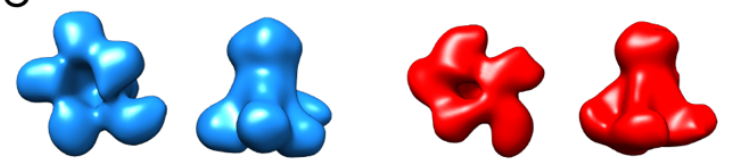

Figure 3 CorA activity in $D_{2} \mathrm{O}$ and direct visualization by negative stain EM. A: CorA activity in the conditions used for SANS. The traces are the normalized fluorescence signals after adding $\mathrm{Mg}^{2+}$ to either empty POPC liposomes (Mock), CorA-POPC proteoliposomes (CorA), or CorA$P O P C$ proteoliposomes preincubated with the inhibitor $\mathrm{Co}\left[\mathrm{NH}_{3}\right]^{3+}$ in $\mathrm{D}_{2} \mathrm{O}(\mathrm{CorA}+\mathrm{CoHex})$. B: Negative stain EM of CorA in DDM and $\mathrm{D}_{2} \mathrm{O}$ with and without $\mathrm{Mg}^{2+}$. The 25 most abundant $2 \mathrm{D}$ classes are shown for each condition. The box dimensions are $170 \times 170 \AA^{2}$ for scale. C: Final $3 D$ model for each condition shown from the intracellular side and in side-view, respectively.

\section{Model refinement to SANS data shows that CorA is asymmetric}

The SANS data sets obtained in SDDM (Figure 2A) exhibit well-defined Guinier-regions and the calculated radii of gyration, $\mathrm{R}_{\mathrm{g}}$, of $42.1 \pm 1.3 \AA\left(+\mathrm{Mg}^{2+}\right)$ and $43.8 \pm 1.7 \AA\left(-\mathrm{Mg}^{2+}\right)$ are close to the predicted values from the X-ray $(41.3 \AA)$ and cryo-EM structures (42.2 $\AA$ and $42.3 \AA$ ). Thus, these data are indicative of well-separated CorA pentamers with no interference from visible lipids or the kind, providing the optimal basis for structural modelling. Given the identicality of the SANS data obtained on the samples of CorA in SDDM with and without $\mathrm{Mg}^{2+}$, we performed structural modeling on only a single data set, that is CorA in SDDM without $\mathrm{Mg}^{2+}$ (Figure 2A, red). Despite controversies on the open state, there is consensus that the 
crystallized symmetric state represents the closed state of the protein. Surprisingly, we could not obtain good fits of the symmetric state to our SANS data without clear systematic deviations, especially at the feature present at $q \approx 0.1 \AA^{-1}$ (Figure $4 A, 4 I 0 U$ ). This was also the case for the asymmetric cryo-EM structure (Figure 4A, 3JCG) that produced an even worse fit. In SANS, the signal represents a population-weighted average of all conformations that the protein can adopt. With a measurement time on the order of several minutes and illumination of $\approx 10^{12}-10^{13}$ molecules, all accessible populations are expected to contribute to the signal. A relatively flat energy landscape with multiple interconverting states has been proposed in $\mathrm{Mg}^{2+}$-free conditions, making a fit of a single structure less meaningful in this context. However, it is unlikely that the average of an ensemble of asymmetric structures give rise to the same SANS signal as that of a single symmetric state corresponding to the structure determined by crystallography and cryo-EM in high $\mathrm{Mg}^{2+}$.

With no scattering contribution from the carrier systems, it becomes possible to analyse the SANS data by conventional methods for soluble proteins, such as bead-modeling. When imposing P5 symmetry, we could obtain envelopes reminiscent of the CorA structure by beadmodelling (Figure 4-figure supplement 1B), whereas no symmetry (P1) imposed lead to asymmetric mass distributions that were not at all compatible with the overall architecture of CorA (Figure 4-figure supplement $1 \mathrm{~A}$ ). This indicates that there is significant structural dispersion in the sample.

To obtain a molecular constrained model compatible with the data, we applied a modified type of normal mode analysis (NMA), starting from the closed crystal structure. A structure with mostly intact secondary structure but a high degree of asymmetry in the ICDs yielded a great fit to the SANS data (Figure 4B). Importantly, this model describes the feature at $q \approx 0.1 \AA^{-1}$, where the PDB models deviate the most. Thus, on average, the solution structure of CorA appears to be asymmetric, in line with our EM models (Figure $3 \mathrm{C}$ ). Again, we emphasize that such an overall asymmetric structure of CorA in presence of excess $\mathrm{Mg}^{2+}$ is in stark contrast to the picture of a closed, symmetric structure that has served the basis for all proposed mechanisms of $\mathrm{Mg}^{2+}$ gating. However, a single asymmetric model as derived from NMA (Figure $4 \mathrm{~B}$ ) is neither compatible with a single set of peaks in NMR (Figure 2C and Figure 2E) nor the substantial experimental evidence for a nearly symmetric, closed state in presence of $\mathrm{Mg}^{2+}$. Likely, CorA adopts multiple different states ${ }^{20,23}$, and according to our data does so both with and without $\mathrm{Mg}^{2+}$ bound. In this case, the SANS data would represent the number weighted average of different states that must be overall asymmetric. 
To model the apparent asymmetry in CorA in more detail, we performed coarse grained molecular dynamics simulations (MD). First, we set up CorA embedded in a POPC bilayer using the Martini3.0b force field. Starting from the symmetric or asymmetric structures, $32 \mu \mathrm{s}$ and $20 \mu$ s simulations, respectively, without any inter-chain elastic network terms yielded only small structural fluctuations (data not shown), which did not significantly improve fits to the experimental SANS data, especially not around the feature at $q \approx 0.1 \AA^{-1}$. Thus, we extended the analysis to metadynamics simulations (MetaD) that allows for enhanced sampling of structural dynamics. MetaD drives the simulation towards a larger variety of structural states based on an energetic bias on a structural feature, a so-called collective variable, here the $R_{g}$ on specific ICD residues. Starting from the symmetric structure, the MetaD simulation quickly drove the simulation away from the local structural minimum that the standard MD had been trapped in and sampled a large range of conformationally different structures (Figure 4C).

The averaged back-calculated SANS of the entire ensemble of structures obtained from MetaD simulations did not fit the experimental SANS data satisfactorily (data not shown). This can be explained by inaccuracies in the simulation that arise from e.g. inaccuracies in the coarse grained force field and imprecisions in the simulation that in turn arise from e.g. insufficient sampling. To resolve this, we applied the Bayesian/Maximum Entropy reweighting method (BME) to optimize the weights of the individual conformations in the simulation with the aim of obtaining an ensemble in better agreement with the experimental SANS data. In addition to the BME, we enforced that a symmetric state should be present in the final ensemble, given the substantial experimental evidence for this state in the literature and that it was under-sampled in the simulation. The best fit to the SANS data (Figure 4D) was obtained with an ensemble consisting of $40 \% \pm 28 \%$ symmetric CorA and the remainder of asymmetric conformations from the MetaD (Figure 4E and Figure 4-figure supplement 3 ). Despite some systematic deviations, the fit is much improved with regards to describing the feature in the SANS data at $q \approx 0.1 \AA^{-1}$, as compared to the fits obtained with the symmetric crystal structure or the open cryo-EM structures, respectively (Figure 4A).

To visualise the results of the MetaD simulation and hence the simulated dynamics, we cluster similar structures and show the four most predominant cluster centroids (Figure 4E) where especially one cluster predominate in the final fitted ensemble. Although the four cluster centroids are wide apart in simulation time $(\approx 500 \mathrm{~ns})$, they are structurally similar with a maximum pairwise RMSD of $6.5 \AA$ (data not shown). The main difference is the distance between the two protomers (Figure 4E, black arrow), which indicate that the individual domains of the ICD can move relative to each other. This is in line with the subunit 
displacements described in the symmetry-break based gating model derived from the cryoEM structures and supported by AFM measurements. Importantly, however, we find that these movements occur irrespective of the presence of $\mathrm{Mg}^{2+}$. Interestingly, CorA mutants were recently shown to crystallize in the symmetric, closed state without $\mathrm{Mg}^{2+}$ bound at the regulatory M1 sites. While the channel is surely closed in the presence of high $\mathrm{Mg}^{2+}$, this observation challenges the claim that release of interfacial bound $\mathrm{Mg}^{2+}$ is the cause for symmetry-break and opening of the channel at low $\mathrm{Mg}^{2+}$ levels. In light of this, our data suggest that CorA could also be in an asymmetric, closed state at high $\mathrm{Mg}^{2+}$ levels. Indeed, we find that the structure of CorA is better described as a distribution of symmetric and asymmetric structures with no large-scale conformational differences between the open and closed states.

A

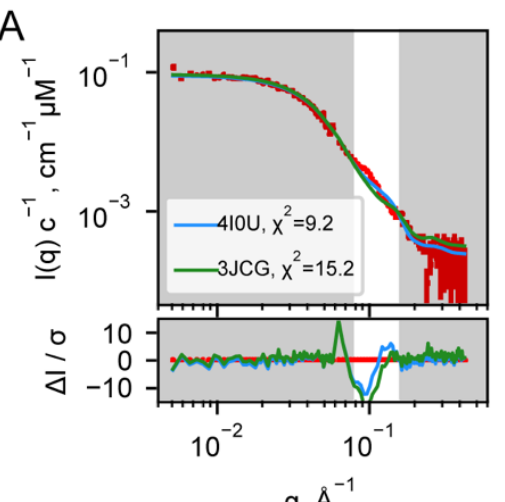

$\mathrm{q}, \AA^{-1}$

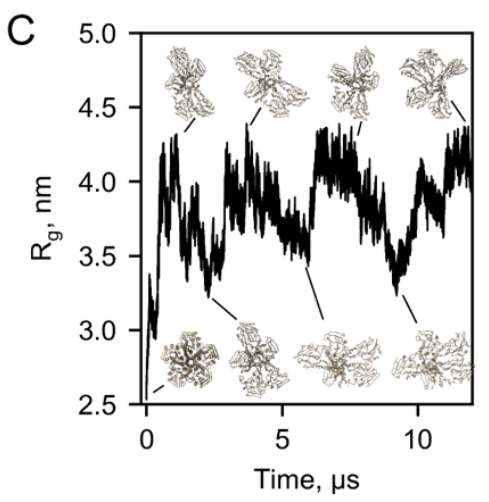

E

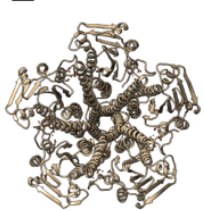

$40 \%$

Input

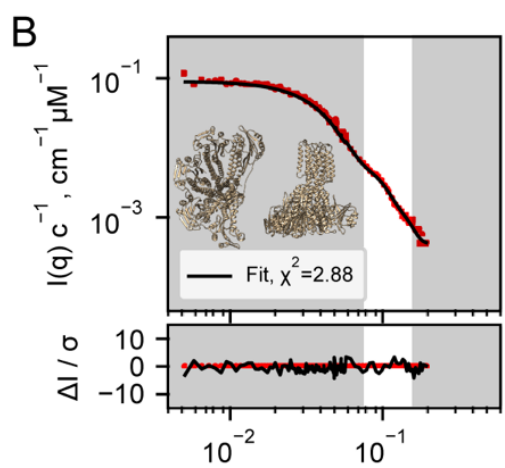

q, $\AA^{-1}$

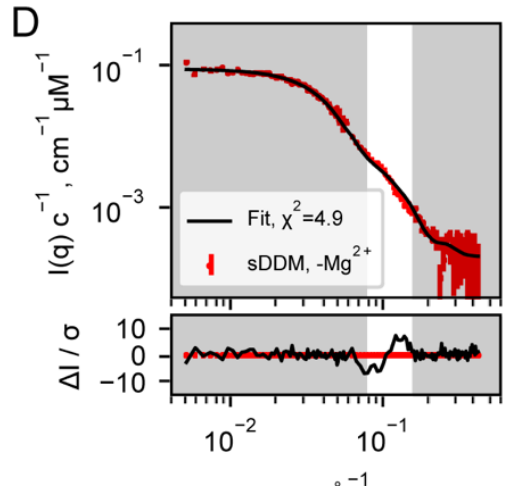

q, $\AA^{-1}$

344 Figure 4 Structural modeling of CorA from SANS data. A: Model fits of the closed crystal structure (4IOU) and open cryo-EM structure (3JCG) to the experimental SANS data obtained in sDDM without $\mathrm{Mg}^{2+}$. The bottom panel shows the error-normalised difference plot of the fits. $B$ : Model fit of the structure obtained by regularised normal mode analysis (bottom and side views inserted on plot). C: MetaD molecular dynamics trajectory with representative frames visualized as ribbon structures. D: Fit of a weighted ensemble obtained from the MD simulation 
in B together with a $40 \%$ contribution from the symmetric CorA. E: The 4 highest weighted cluster centroids in the metaD simulation (right bracket) together with the closed symmetric structure (left) which together illustrates the ensemble of CorA structures that produced the best fit to the experimental SANS data.

Figure 4-figure supplement 1 Bead modeling of CorA. All four SANS data sets were analysed by the DAMMIF pipeline ${ }^{38}$ available from the ATSAS package ${ }^{39}$. In total, DAMMIF was run 100 times in slow mode with either P1 (A) or P5 (B) symmetry selected. For P1 symmetry, the normalized spatial discrepancy (NSD) values varied from 1.2 to 1.5 , whereas for P5 symmetry, NSD values varied from 1.4 to 1.7. NSD values above 1 indicate that the calculated models differ systematically from one another.

Figure 4-figure supplement 2 Goodness of fit from NMA generated structures to SANS. The best compromise between conservation of structure and the best fit to the data is in the "elbow"-region, here chosen at $S=0.01$ (with $\alpha=600$ ).

Figure 4-figure supplement 3 Ensemble fitting to SANS data with symmetric CorA included. The goodness of fit $\left(\chi^{2}\right)$ plotted as a function $f$, the fraction of $4 I 0 U$, fixed before $B M E$ reweighting of the MetaD generated ensemble to fit the SANS data. The data on the graph were fitted by a quadratic function to yield the minimum with standard deviation at $40 \pm 28 \%$ $410 U$, where the standard deviation was calculated by an increment in $\chi^{2}$ of 1 .

\section{Structural dynamics are different in open and closed states of CorA}

So far, we have considered a set of static snapshots to interpret the wide variety of populated states of CorA. The observation of MAS NMR dynamical probes sheds light on the backbone motions of these states over different timescales, enriching the structural description of CorA with conformational plasticity.

373 A first insight on site-specific dynamics is obtained by comparing the peak intensities observed in the MAS NMR experiments in the two samples with and without $\mathrm{Mg}^{2+}$.

375 Peak intensities are dependent on dipolar couplings between nearby nuclei and are affected when such couplings are averaged by local motions. For an amide ${ }^{1} \mathrm{H}-{ }^{15} \mathrm{~N}$ pair, this corresponds to motional processes faster than $\sim$ tens of $\mathrm{kHz}$ (i.e. more rapid than $\sim$ hundreds of $\mu s)$.

379 Changes in peak intensity are already noticeable in the $2 \mathrm{D}{ }^{1} \mathrm{H}-{ }^{15} \mathrm{~N}$ dipolar correlation spectra, but are amplified in the 3D correlations, where additional radio-frequency irradiation periods act as a more stringent filter, dumping the signals of the most mobile sites. 1D traces of two exemplar 3D ${ }^{1} \mathrm{H}-{ }^{15} \mathrm{~N}-{ }^{13} \mathrm{C}$ correlations (T65 in the ICD and F306 in the TMD) and the plot of signal intensities over the full protein sequence with and without $\mathrm{Mg}^{2+}$ are shown in Figure 5A and Figure 5B, respectively. As mentioned above, an overall decrease in peak intensities is associated to removal of $\mathrm{Mg}^{2+}$, with a stronger effect observed in the TMD. This points toward a variation of the dynamic behavior of this region in the two samples. reporters of motions occurring over a window of hundreds of ns to hundreds of $\mu$ s. These parameters were measured site-specifically for $\sim 40$ residues in CorA backbone, by introducing 
a relaxation filter in the ${ }^{1} \mathrm{H}-{ }^{15} \mathrm{~N}$ dipolar correlation module and monitoring the signal decay of each amide pairs in a series of experiments with increasing relaxation delays (Figure $5 \mathrm{C}$ ). A remarkable (up to 10 fold) increase was observed throughout the entire protein upon removal of $\mathrm{Mg}^{2+}$ (Figure 5D). The same effect was observed for the ${ }^{15} \mathrm{~N}$-alanine labelled sample (Figure 5 -figure supplement $1 \mathrm{~A}$ ). An additional measurement of ${ }^{15} \mathrm{~N} R_{1 p}$ rates upon a $\sim 20^{\circ} \mathrm{C}$ cooling revealed a different temperature-dependent behaviour of the two $\mathrm{Mg}^{2+}$-loaded samples (Figure 5-figure supplement $1 \mathrm{~B}$ ). While in the presence of $\mathrm{Mg}^{2+}$ relaxation rates were conserved at low temperatures, an important (2-3 fold) decrease was observed in the $\mathrm{Mg}^{2+}$ free form. Finally, also dynamics on the ns timescale obtained by the measurements of bulk backbone ${ }^{15} \mathrm{~N}_{1}$ showed a global increase of relaxation rates (by a factor $\sim 1.5$ ) upon removal of $\mathrm{Mg}^{2+}$ (Figure 5-figure supplement 1C).

In summary, MAS NMR reveals that removal of $\mathrm{Mg}^{2+}$ triggers an increase in the backbone flexibility on different timescales, a dynamical effect which is different for the ICD and the TMD.

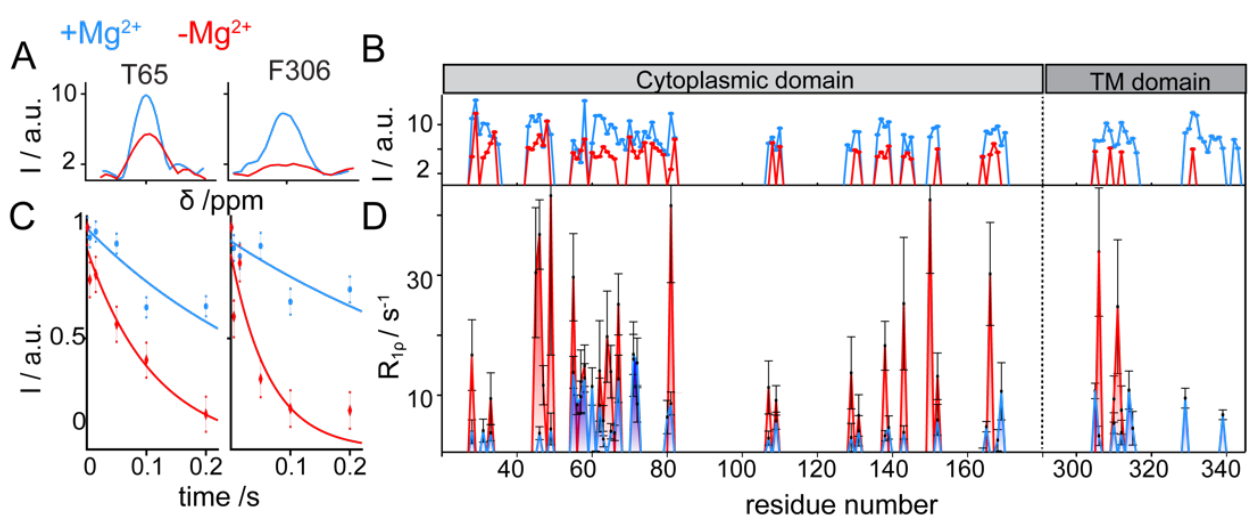

403

404

405

406

407

408

409

410

411

412

413

414

415

416

417

418

Figure 5 CorA backbone dynamics by MAS NMR in DMPC in presence (blue) and in absence (red) of $\mathrm{Mg}^{2+}$ A: Examples of $1 D$ traces of $3 D^{1} \mathrm{H}^{15}{ }^{15}-{ }^{13} \mathrm{C}$ peaks for two residues in the ICD (T65) and in the TMD (F306). B: Comparison of peak intensities in $3 D^{1} \mathrm{H}_{-}{ }^{15} \mathrm{~N}-{ }^{13} \mathrm{C}$ spectra over the protein sequence. ICD and TMD are indicated by boxes of different color on top of the plot. $C-D$ : Site-specific ${ }^{15} \mathrm{~N} R_{1 \rho}$ rates measured with a $15 \mathrm{kHz}$ spin-lock field. C: Examples of ${ }^{15} \mathrm{~N}$ $R_{1 \rho}$ relaxation decays together with the corresponding mono-exponential fits for residues T65 and F306. D: Comparison of site-specific backbone ${ }^{15} \mathrm{~N} R_{1 \rho}$ rates plotted along the CorA sequence.

Figure 5-figure supplement 1 CorA backbone dynamics by MAS NMR: Site-specificity and temperature dependence. A: Site-specific ${ }^{15} \mathrm{~N} R_{1 \rho}$ rates measured with a $15 \mathrm{kHz}$ spin-lock field in the ${ }^{15} \mathrm{~N}$-Ala sample of CorA. B: Comparison of site-specific ${ }^{15} \mathrm{~N} R_{1 \rho}$ rates measured with a $15 \mathrm{kHz}$ spin-lock field at $280 \mathrm{~K}$ in the presence (cyan) and in the absence (magenta) of $\mathrm{Mg}^{2+}$. Dashed lines indicate the average relaxation rate values in the ICD and TMD distinctly, at higher (blue and red) and lower (cyan and magenta) temperature. C: Bulk ${ }^{15} N R_{1}$ decay curves measured at $300 \mathrm{~K}$. Experimental curves were fitted with a biexponential decay.

420 The functional mechanism of the pentameric divalent cation channel CorA of Thermotoga 421 maritima has been under investigation since the release of the first high resolution structures 
in 2006. It is now well acknowledged that gating involves a conformational transition between

a closed symmetric state and one or many asymmetric states. The concise current model derived from cryo-EM ${ }^{20}$ and $\mathrm{HS}-\mathrm{AFM}^{23}$ defines a rigid symmetric conformation at high $\mathrm{Mg}^{2+}$ concentrations (>4 mM), dynamic asymmetric conformations at low $\mathrm{Mg}^{2+}$ concentrations (2-3 $\mathrm{mM}$ ) and several distinct rigid asymmetric conformations in the absence of $\mathrm{Mg}^{2+}$.

We have performed SANS in invisible carrier systems to study the solution conformation of CorA, together with modelling of both static and ensemble structures obtained from MetaD simulations. Furthermore, we used ${ }^{1} \mathrm{H}$-detected solid-state NMR to investigate CorA conformation and dynamics in hydrated lipid bilayers. These complementary methods allow us to expand the current view on the mechanistically important conformational equilibria.

Asymmetric states are populated in CorA at high $\mathbf{~ M g}^{2+}$ concentrations. Crystal structures of CorA from Thermotoga maritima (Tm) and Methanocaldococcus jannaschii (Mj) solved in the $\mathrm{Mg}^{2+}$-bound state revealed a symmetric bell-like structure, showing a narrow inner channel, representing the closed form. All proposed gating models were built on the common basis of a single rigid and symmetric conformation at high $\mathrm{Mg}^{2+}$ concentrations ${ }^{11,12,16,20,23,40,41}$. Our SANS data recorded on CorA in high $\mathrm{Mg}^{2+}$ concentrations is inconsistent with a single symmetric structure and can only be explained by symmetry breaking and/or a mixture of populations e.g. through the concomitant presence of symmetric and asymmetric states. Indirect indications for a mixture of populations even in the presence of $\mathrm{Mg}^{2+}$ can be found in the literature: For example, the symmetric cryo-EM structure (3JCF) obtained at high $\mathrm{Mg}^{2+}$ concentrations, resulted from roughly $60 \%$ of the particles ${ }^{20}$, which implies that asymmetric states could partly account for the remaining 40\%. Although with lower resolution, our negative-stain TEM data indeed support the presence of such populations. HS-AFM measurements ${ }^{23}$ showed that the symmetric, closed state was stable in starting conditions at high $\mathrm{Mg}^{2+}$. However, only $35 \%$ of this state was recovered after complete removal and reintroduction of $\mathrm{Mg}^{2+}$ ions, suggesting secondary effects working on adjusting the equilibrium between fluctuating asymmetric states and the closed symmetric state.

A symmetric state is populated in CorA in absence of $\mathbf{M g}^{2+}$. The conformation of metalfree CorA has been the object of a long ongoing speculation since wild-type CorA failed to crystallize in the absence of $\mathrm{Mg}^{2+}$. Asymmetric subunit arrangements were first proposed based on an $X$ - ray structure of a truncated CorA variant $(\Delta N 25 / R 222 A / K 223 A)^{12}$. Since the release of two cryo-EM structures of wild-type $\operatorname{CorA}^{20}$, it is now a common belief that this channel adopts exclusively an asymmetric arrangement in absence of $\mathrm{Mg}^{2+}$. 
spectra do not change significantly upon removal of $\mathrm{Mg}^{2+}$ indicates the conservation of local symmetric environments for most of the NMR-active nuclei in both conditions. This evidence in turn suggests that the global symmetry is maintained for a substantial population of CorA pentamers, as it is unlikely that the ample rearrangements implied by the cryo-EM model could occur without affecting local symmetries of so many nuclei in the five subunits. This view is additionally corroborated by our negative-stain TEM data, which showed the presence of nearly symmetric $2 \mathrm{D}$ classes in $\mathrm{Mg}^{2+}$-free preparations.

Symmetry breaking is expected to result in local conformational heterogeneities between the monomers, resulting in peak splitting, with a possible reduction of the intensity beyond the detection limit. In this regard, the general decrease in intensity of the NMR spectra observed in absence of $\mathrm{Mg}^{2+}$ suggests a significant reduction of the population of the observed symmetric state, compatible with the co-existence of a broad distribution of asymmetric states. It has previously been shown that CorA mutants with abolished M1 regulatory sites still crystallize in a symmetric, closed state. This suggests that binding of $\mathrm{Mg}^{2+}$ is not necessary for closing the channel ${ }^{19}$.

474 The two asymmetric cryo-EM structures were refined from only about $15 \%$ each of the picked particles, indicating the presence of various conformations, and possibly the symmetric form. AFM provided a more detailed conformational analysis and found around $20 \%$ symmetric structures at low $\mathrm{Mg}^{2+}$ concentrations (0-3 mM). The closely related $\mathrm{Zn}$ transporter $\mathrm{ZntB}$ also showed a symmetric structure in the absence of regulatory cations ${ }^{42}$. All these observations point towards a conformational equilibrium between the symmetric and various asymmetric states which is partially but not entirely shifted towards asymmetric forms at low cation concentration.

Increased dynamics in CorA in absence of $\mathbf{M g}^{2+}$. A "dynamic character" of CorA at low levels of $\mathrm{Mg}^{2+}$ was postulated on the bases of the cryo-EM data ${ }^{20}$ and recently characterized by high-speed $\mathrm{AFM}^{23}$. An elevated conformational plasticity would indeed be consistent with the hitherto unsuccessful crystallization of a conducting state. MAS NMR has the exclusive advantage of probing molecular motions with site-specific resolution. Differently from a previous NMR study on a truncated construct in detergent micelles ${ }^{43}$, we here directly tackled the full-length protein in lipid bilayers. Notably, we characterized the change in dynamics occurring upon $\mathrm{Mg}^{2+}$ release by acquiring $\mathrm{NMR}$ observables sensitive to different timescales. On a fast ps-ns timescale, increased bulk ${ }^{15} \mathrm{~N}$ $\mathrm{R}_{1} \mathrm{~S}$ in the absence of $\mathrm{Mg}^{2+}$ are indicative of less restricted backbone motions, as also suggested by MD simulations ${ }^{41}$. Site-specific ${ }^{15} \mathrm{~N} \mathrm{R}_{1 \rho}$ rates are reporters of slower segmental motions displacing secondary structure elements with respect to each other, over a window of 
hundreds of ns to hundreds of $\mu \mathrm{s}$. These dynamical processes appear to be largely promoted over the whole CorA structure when $\mathrm{Mg}^{2+}$ is absent.

Notably, a change in dynamics is also visible in the TMD region. Here we detected and assigned most trans-membrane and periplasmic residues including the GMN motif in the presence of $\mathrm{Mg}^{2+}$. However, for these residues, peaks broadened beyond detection when $\mathrm{Mg}^{2+}$ was removed. This points towards the presence of multiple conformations in this region as well, although the exchange dynamics likely occurs on a different regime with respect to the ICD domain. An earlier work suggested that removal of $\mathrm{Mg}^{2+}$ results in a combination of lateral and radial tilting of two adjacent monomers, which allows the creation of interactions between them $^{12}$. This idea was recently extended based on coarse-grained MD simulations that show multi-step conformational changes propagating from the ICD to the TMD helices ${ }^{22}$. In line with these observations, we propose that collective ns- $\mu$ s motions of the backbone initially promoted in the ICD by release of $\mathrm{Mg}^{2+}$ ions would in turn induce higher conformational flexibility in the TMD.

\section{Are all asymmetric states open/conductive? What is an open/conductive state? Since} asymmetric structures are present both in presence and absence of $\mathrm{Mg}^{2+}$, lack of symmetry is not equivalent to conduction. The regulation of $\mathrm{Mg}^{2+}$ transport clearly needs to be dependent on more stringent control. Interestingly, a previous MD study ${ }^{21}$ found that dry and intermediate transiently wetted states, both non-conducting, were present both with and without bound $\mathrm{Mg}^{2+}$ and interconversion rates on the ns time scale. Furthermore, in absence of $\mathrm{Mg}^{2+}$, a conducting "stably superhydrated" state was sampled, i.e. where the region of pore lining residues M291, L294, A298, and M302 that form the main hydrophobic constriction is wetted by more than 20 water molecules. Though this state was only sampled in a minor part of the simulations, its population could change at a longer time scale. Indeed, the ns sampled by the simulations cannot describe our observations that CorA adopts symmetric and asymmetric conformations, both in presence and absence of $\mathrm{Mg}^{2+}$, while in absence of $\mathrm{Mg}^{2+}$, the channel has higher motional freedom on the $\mu$ s timescale. In light of the MD results, our combined findings support the idea that the "stably superhydrated" state is present in low abundance and interconverts rapidly with the remaining ensemble of states.

An integrated view on CorA transport regulation. Our findings cannot be explained in terms of sharp transitions between open and closed states. An alternative model that integrates our findings with the previously available literature is schematically illustrated in Figure 6. CorA samples symmetric and asymmetric conformational states, whose distribution is tuned by the $\mathrm{Mg}^{2+}$ concentration. Between the symmetric and open states, a relatively flat energy landscape exists with asymmetric, closed states, both in the presence and absence of $\mathrm{Mg}^{2+}$. Low $\mathrm{Mg}^{2+}$ 
intracellular levels induce a reduction in the population of the symmetric, closed state together with a decrease of the energy barrier towards the open state, which becomes more populated. At these $\mathrm{Mg}^{2+}$ concentrations, CorA can visit a conducting asymmetric ensemble of states. In this context, increased dynamics, as observed by NMR, can become the key determinant allowing CorA to explore different wells of the energy profile, making the open state reachable.

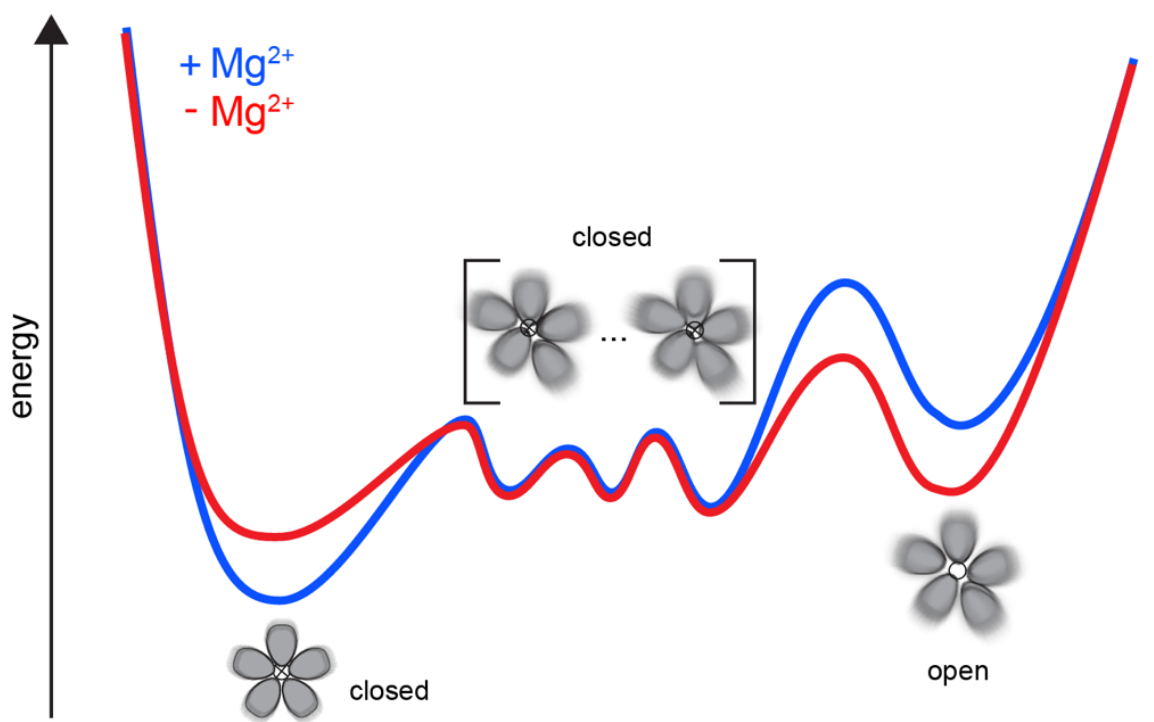

Figure 6 Schematic representations of a dynamic model for CorA. A complex landscape connects a symmetric and closed state with an asymmetric open state, both in the presence and in the absence of regulatory ions.

Such a dynamic model is equally compatible with previously unexplainable symmetric crystal structures of M1-binding site mutants in the absence of $\mathrm{Mg}^{2+19}$. While for WT CorA, removal of $\mathrm{Mg}^{2+}$ increases the dynamics and shifts the conformational equilibria, point mutations probably have an opposite effect, stabilizing the symmetric state and allowing crystal formation.

Relating to other classes of proteins and channels. Gating has been investigated extensively in the family of pentameric ligand-gated ion channels (pLGIC) ${ }^{44}$, which in general have symmetric or pseudo-symmetric structures in the ligand-free form. Intriguingly, the existence of asymmetric conformations has been observed in the absence of ligand for the serotonine receptor $5 \mathrm{HT} 3 \mathrm{R}$, revealing conformational plasticity also in the resting state. Such conformations are more energetically favorable when the agonist is bound, suggesting an asymmetric activation where resting asymmetric states are possible intermediates of the gating ${ }^{45}$. Asymmetric activation was proposed also for the nicotinic receptor nAChR, where the movement of pore lining helices was shown to induce the asymmetry in the ligand-bound form ${ }^{46}$. Taken together, these studies suggest that asymmetry might have a more widespread occurrence in channel regulation. Asymmetric resting states may exist as intermediates in the 
conformational landscape independently of the presence of substrate, but they become more energetically favorable when this is bound.

\section{Conclusion}

562 The availability of room temperature SANS and NMR data of CorA in lipid bilayers, reporting

563 on both global and local behaviour of this channel, supported by MD simulations, allowed us

564 to extend and rationalise the "symmetry-break-upon-gating" model for $\mathrm{Mg}^{2+}$ transport. Our

565 observations support the suggestion that asymmetric conformations are involved in the gating 566 mechanism, but in a more complex way than a simpler two-state picture, where $\mathrm{Mg}^{2+}$ bound 567 CorA is a stable, symmetric structure. Indeed, we find that the CorA pentamer is a symmetry568 broken fluctuating structure able to explore a wide conformational landscape both in the 569 presence and absence of $\mathrm{Mg}^{2+}$. We propose that the determining factor for CorA to visit 570 conducting states is the increase in backbone flexibility on different timescales upon release 571 of regulatory $\mathrm{Mg}^{2+}$. Future investigations of the conformational equilibria will enrich the insight 572 provided in this study with a better mechanistic understanding of the kinetics and 573 thermodynamics of gating and transport.

574 Materials and methods

\section{Materials}

576 All chemicals were from Sigma-Aldrich unless otherwise stated. DDM was from Carbosynth

577 (UK), match-out-deuterated DDM $\left(\mathrm{sDDM}^{29}\right)$ and match-out deuterated POPC (d-POPC) were 578 synthesised at the National Deuteration Facility at ANSTO (Lucas Heights, Australia). The d579 POPC was synthesised as previously ${ }^{47}$, but with custom deuteration (94\% D in tail groups, 71 $580 \% \mathrm{D}$ in head group). Details of the synthesis and chemical and isotopic analysis is described 581 below.

582

\section{Protein production and purification}

584 For SANS, CorA was produced and purified essentially as described elsewhere ${ }^{48}$. For studies 585 in DDM, the N-terminal His-tag was cleaved by tobacco etch virus (TEV) protease before gel 586 filtration, whereas for nanodiscs, it was cleaved after incorporation (see below). For NMR, 587 uniformly isotopically labelled CorA was produced in $\mathrm{M} 9$ medium containing $3 \mathrm{~g} / \mathrm{L}{ }^{13} \mathrm{C}$-glucose 588 and $1 \mathrm{~g} / \mathrm{L}{ }^{15} \mathrm{~N}$-ammonium chloride. ${ }^{15} \mathrm{~N}$-alanine labelled CorA was produced in $\mathrm{M} 9$ medium 589 containing regular ${ }^{12} \mathrm{C}$-glucose and ${ }^{14} \mathrm{~N}$-ammonium chloride and supplemented with $200 \mathrm{mg} / \mathrm{L}$ 590 of ${ }^{15} \mathrm{~N}$-alanine. Match-out deuterated circularised membrane scaffold protein (MSP), d- 
591 csMSP1E3D1, was produced at the D-lab at ILL (Grenoble, France) and purified as described

592 previously ${ }^{48}$. Proteins were stored at $-80^{\circ} \mathrm{C}$ until used.

593

594 Synthesis of match-out deuterated POPC

595 The overall synthesis of POPC- $d_{77}$ is reported elsewhere ${ }^{47}$. Figure 7 shows the synthetic 596 scheme followed in this study to produce the specific level of deuteration in the head and tail 597 groups of the POPC. The specific level of deuteration in the tail group was achieved by diluting 598 pure heavy water with light water in specific ratios in the Parr reactor when making the 599 deuterated alkyl chains from their fatty acid precursors ${ }^{29}$. The analysis data and spectra of the 600 intermediate compounds and the final compound are shown in Figure 7-figure supplements 1 601 to 23. Electrospray ionisation mass spectra (ESI-MS) were recorded on a 4000 QTrap AB 602 SCIEX Mass Spectrometer. The overall percent deuteration of the molecules was calculated 603 by ER-MS (enhanced resolution - MS) using the isotope distribution analysis of the different 604 isotopologues by analysing the area under each MS peak which corresponds to a defined 605 number of deuterium atoms. The contribution of the carbon-13 (natural abundance) to the 606 value of the area under each $[X+1]$ MS signal is subtracted based on the relative amount found 607 in the protonated version. In a typical analysis we measure the C-13 natural abundance 608 contribution by running ER-MS of the protonated version (or estimate it by ChemDraw 609 software) and use this value in our calculation using an in-house developed method that 610 subtracts this contribution from each MS signal constituting the isotope distribution. ${ }^{1} \mathrm{H}$ NMR 611 (400 MHz), ${ }^{13} \mathrm{C}$ NMR (100 MHz), ${ }^{31} \mathrm{P}$ NMR (162 MHz) and ${ }^{2} \mathrm{H}$ NMR $(61.4 \mathrm{MHz})$ spectra were 612 recorded on a Bruker $400 \mathrm{MHz}$ spectrometer at $298 \mathrm{~K}$. Chemical shifts, in ppm, were 613 referenced to the residual signal of the corresponding solvent. Deuterium NMR spectroscopy 614 was performed using the probe's lock channel for direct observation. 


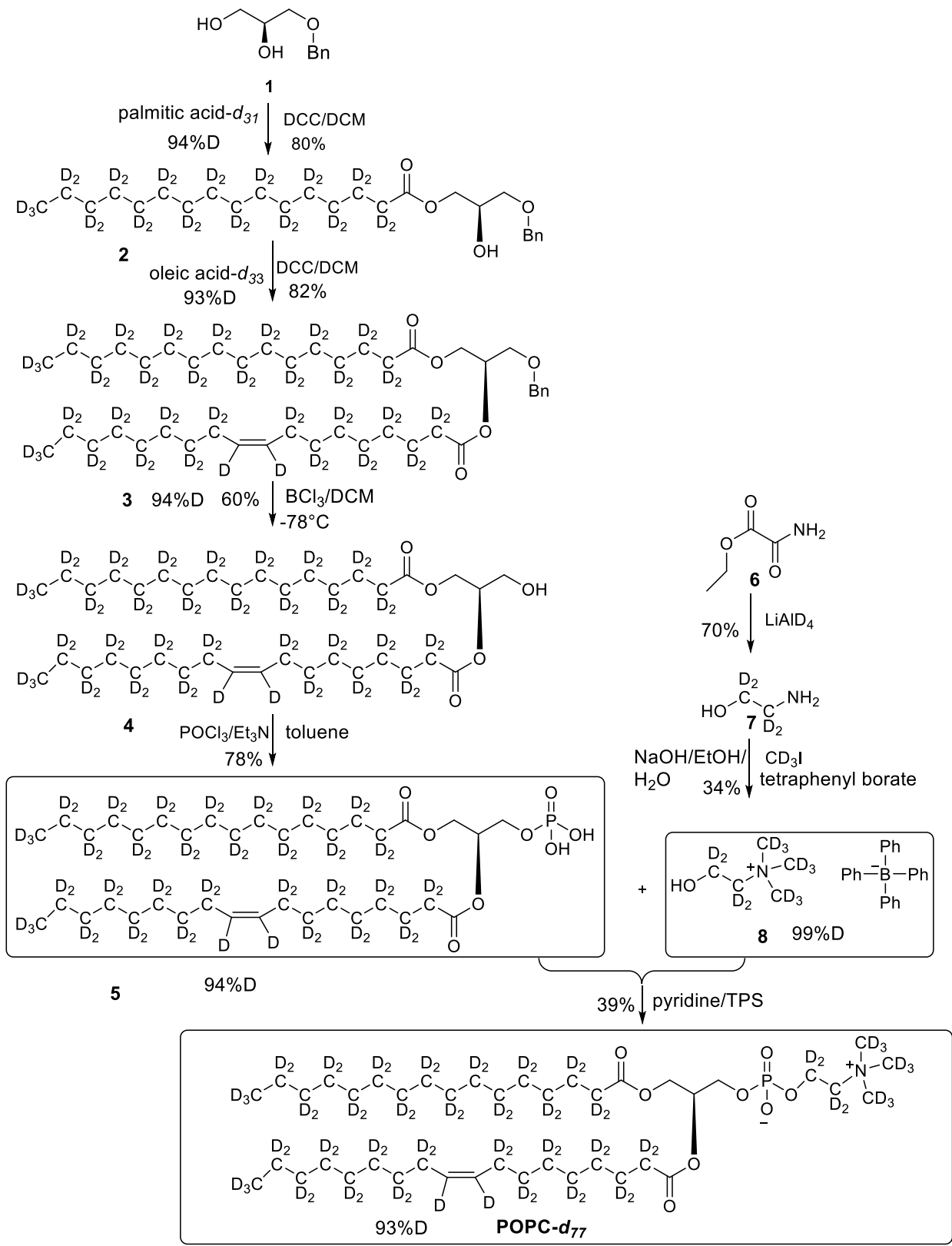

Figure 7 Overall synthesis achieved by following a reported paper, all the intermediates and 618 final POPC- $d_{77}$ were obtained in similar yields ${ }^{47}$.

619 Figure supplement $1^{1} \mathrm{H}$ NMR of 1-palmitoyl-d $d_{31}$-sn-3-benzyloxy-glycerol (Figure 7, molecule 620 2) in $\mathrm{CDCl}_{3}$. (400 MHz, $\left.\mathrm{CDCl}_{3}\right)$, $\delta$ residual protons $0.88(\mathrm{~m}, 0.22 \mathrm{H}), 1.10-17(\mathrm{~m}, 1.96 \mathrm{H})$, $621 \quad 1.57 .1 .86(\mathrm{~m}, 1.55 \mathrm{H}), 1.96(\mathrm{~m}, 0.54 \mathrm{H}), 3.69(\mathrm{~m}, 2 \mathrm{H}), 4.09(\mathrm{~m}, 1 \mathrm{H}), 4.22(\mathrm{~m}, 2 \mathrm{H}) 4.60(\mathrm{~s}, 2 \mathrm{H})$, $6227.36(m, 5 H)$.

623 Figure supplement $2^{2} \mathrm{H}$ NMR of 1-palmitoyl-d ${ }_{31}$-sn-3-benzyloxy-glycerol (Figure 7, molecule 624 2) in $\mathrm{CDCl}_{3 .}\left(400 \mathrm{MHz}, \mathrm{CDCl}_{3}\right), \delta 0.82(\mathrm{~m}), 1.18(\mathrm{~m}), 1.54(\mathrm{~m}), 2.27(\mathrm{~m})$.

625 Figure supplement $3{ }^{13} \mathrm{C}$ NMR of 1-palmitoyl-d $d_{31}$-sn-3-benzyloxy-glycerol (Figure 7, molecule 626 2) in $\mathrm{CDCl}_{3} .\left(400 \mathrm{MHz}, \mathrm{CDCl}_{3}\right), 10.9(\mathrm{~m}), 22.09(\mathrm{~m}), 28.33(\mathrm{~m}), 33.05(\mathrm{~m}), 65.33$, 68.9, 70.80,

628 Figure supplement $4{ }^{1} H$ NMR of 1-palmitoyl-d $d_{31}-2$-oleoyl-d $d_{33}$-sn-3-benzyloxy-glycerol (Figure 629 7, molecule 3) in $\mathrm{CDCl}_{3}$. (400 MHz, $\left.C D \mathrm{Cl}_{3}\right)$, $\delta$ residual protons $0.90(\mathrm{~m}, 3.56 \mathrm{H}), 1.29(\mathrm{~m}$, $6305.15 H), 1.98(m, 0.68 H), 2.29(m, 1.25 H), 3.61(d, J=5.0 H z, 2 H), 4.21(m, 1 H), 4.36(m, 1 H)$, 
protonated benzyl protons $4.56(A B q, J=12 H z, 2 H), 5.26(m, 1 H), 5.35(s, 0.65 H), 7.34(m$, $5 H)$.

633

Figure supplement $5^{2} \mathrm{H}$ NMR of 1-palmitoyl-d $d_{31}-2$-oleoyl-d d3 $_{33}-\mathrm{sn}$-3-benzyloxy-glycerol (Figure 7, molecule 3) in $\mathrm{CDCl}_{3} .(400 \mathrm{MHz}, \mathrm{CDCl}$ ), $\delta 0.82$ ( $\mathrm{m}, 6 \mathrm{D}), 1.19(\mathrm{~m}, 35.35 \mathrm{D}), 1.53$ (m, 3.74D), $1.94(m, 3.34 D), 2.25(m, 2.63 D), 3.56(m, 1.71 D), 4.27(m, 1.04 D), 5.35(m, 1.92)$

Figure supplement $6{ }^{13} \mathrm{C} N \mathrm{NMR}$ of 1-palmitoyl-d $d_{31}$-2-oleoyl-d $d_{33}$-sn-3-benzyloxy-glycerol (Figure 7, molecule 3) in $\mathrm{CDCl}_{3} .\left(400 \mathrm{MHz}, \mathrm{CDCl}_{3}\right), \delta 13.08(\mathrm{~m}), 21.60(\mathrm{~m}), 23.96(\mathrm{~m}), 26.50$ $(\mathrm{m}), 28.2(\mathrm{~m}), 30.60(\mathrm{~m}), 33.80(\mathrm{~m}), 62.60,68.39$ 9, 70.10, 73.1, 127.7, 127.9, 128.5, 137.7, 173.1, 173.4 .

Figure supplement 7 ESI-MS, m/z $749\left[\mathrm{M}^{+1}\right]^{+}$of POPC precursor 1-palmtoyl-2-oleoyl-snbenzyloxyglycerol- $d_{64}$ (Figure 7 , molecule 3). Overall $94 \% D$, isotope distribution $d_{64}, 7.8 \%, d_{63}$, $15.1 \%, d_{62}, 19.5 \%, d_{61}, 18.0 \%, d_{60}, 13.1 \%, d_{59}, 10.2 \%, d_{58}, 6.5 \%, d_{57}, 3.9 \%, d_{56}, 2.7 \%$.

Figure supplement $8{ }^{1} \mathrm{H}$ NMR of 1-palmitoyl-d $d_{31}$-2-oleoyl-d $d_{33}$-sn-3-glycerol (Figure 7, molecule 4) in $\mathrm{CDCl}_{3} .(400 \mathrm{MHz}, \mathrm{CDCl} 3)$, $\delta$ residual protons $0.24(\mathrm{~m}, 0.47 \mathrm{H}), 1.28(\mathrm{~m}, 1.51 \mathrm{H})$, $1.67(\mathrm{~m}, 2.24 H), 2.13(\mathrm{~m}, 0.52 \mathrm{H}), 2.23(\mathrm{~m}, 1.29 H), 3.72(\mathrm{~m}, 2 \mathrm{H}), 4.30(\mathrm{~m}, 2 \mathrm{H}), 5.10(\mathrm{~m}, 1 \mathrm{H})$, $5.34(\mathrm{~s}, 0.48 \mathrm{H})$.

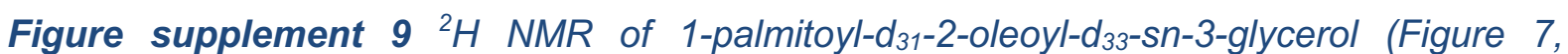
molecule 4) in $\mathrm{CDCl}_{3}$. (400 MHz, $\left.\mathrm{CDCl}_{3}\right), \delta 0.82$ ( $\left.\mathrm{m}, 8.6 \mathrm{D}\right), 1.19$ (m, 49.2D), 1.55 (m, 4.8D), $1.94(m, 3.46 D), 2.28(m, 3.82 D), 5.38(m, 1.1 D)$.

Figure supplement $10{ }^{13} \mathrm{C} N M R$ of 1-palmitoyl-d $d_{31}$-2-oleoyl-d $d_{33}$-sn-3-glycerol (Figure 7, molecule 4) in $\mathrm{CDCl}_{3}$. (400 MHz, $\left.\mathrm{CDCl}_{3}\right), \delta 12.89(\mathrm{~m}), 21.39(\mathrm{~m}), 22.6(\mathrm{~m}), 24.00(\mathrm{~m}), 26.25$ $(\mathrm{m}), 28.2(\mathrm{~m}), 30.49(\mathrm{~m}), 33.86(\mathrm{~m}), 65.00,60.76,61.60,71.64,128.02,129.14,129.5,173.53$, 173.93.

Figure supplement $11{ }^{1} \mathrm{H} N \mathrm{NM}$ of crude product 1-palmitoyl-d $d_{31}$-2-oleoyl-d $d_{33}$-sn-3-glycerophosphatidic acid (Figure 7, molecule 5) in $\mathrm{CDCl}_{3}$. This synthesised crude dried product was used in next step without further purification.

Figure supplement $12{ }^{31} \mathrm{P}$ NMR of crude product 1-palmitoyl-d $d_{31}-2$-oleoyl-d $d_{33}$-sn-3-glycerophosphatidic acid (Figure 7, molecule 5) in $\mathrm{CDCl}_{3}$. This synthesised crude dried product was used in next step without further purification.

Figure supplement 13 ESI-MS, m/z $737\left[\mathrm{M}^{-1}\right]^{\top}$ of crude product of 1-palmitoyl-d ${ }_{31}$-2-oleoyl$d_{33}$-sn-3-glycero-phosphatidic acid (Figure 7, molecule 5). Overall $94 \% D$, isotope distribution $d_{64}, 0.6 \%, d_{63}, 8.9 \%, d_{62}, 18.8 \%, d_{61}, 25.1 \%, d_{60}, 20.6 \%, d_{59}, 13.0 \%, d_{58}, 7.5 \%, d_{57}, 5.2 \%, d_{56}$, $0.2 \%, d_{55}, 0.1 \%$.

Figure supplement $14{ }^{1} \mathrm{H} N M R$ of POPC- $d_{77}$ in $\mathrm{CDCl}_{3 .}$ (400 MHz, CDCl3), $\delta$ residual protons $0.85(\mathrm{~m}, 0.17 \mathrm{H}), 1.25(\mathrm{~m}, 1.48 \mathrm{H}), 1.54(\mathrm{~m}, 0.22 \mathrm{H}), 1.97(\mathrm{~m}, 0.18 \mathrm{H}), 2.22(\mathrm{~m}, 0.66 \mathrm{H}), 3.89(\mathrm{~m}$, $2 H), 4.08(m, 1 H), 4.34(m, 1 H), 5.14(m, 1 H), 5.27(s, 0.43 H)$

Figure supplement $15{ }^{2} \mathrm{H} N M R$ of POPC-d 77 in $\mathrm{CDCl}_{3}$. (400 MHz, CDCl $)_{3}, \delta 0.80(\mathrm{~m}), 1.19$ (m), $2.20(\mathrm{~m}), 1.93(\mathrm{~m}, 6.0 \mathrm{D}), 3.35(\mathrm{~m}), 3.84(\mathrm{~m}), 5.36(\mathrm{~m})$.

Figure supplement $16{ }^{13} \mathrm{C} \mathrm{NMR}$ of POPC- $d_{77}$ in $\mathrm{CDCl}_{3}$. $\left(400 \mathrm{MHz}, \mathrm{CDCl}_{3}\right), \delta 13.03(\mathrm{~m}), 21.48$ $(\mathrm{m}), 23.88(\mathrm{~m}), 26.24(\mathrm{~m}), 28.36(\mathrm{~m}), 30.49(\mathrm{~m}), 33.72(\mathrm{~m}), 53.17,58.76(\mathrm{~m}), 62.24(\mathrm{~m}), 69.84$ (m), $127.90(s), 129.5,173.31,173.69$

Figure supplement $17\left\{{ }^{1} \mathrm{H}\right\}$ and $\left\{{ }^{2} \mathrm{H}\right\}$ decoupled ${ }^{13} \mathrm{C} N M R$ spectra of $\mathrm{POPC}-\mathrm{d}_{77}$ in $\mathrm{CDCl}_{3}$.

Figure supplement $18{ }^{31} \mathrm{P} N M R$ of POPC- $d_{77}$ in $\mathrm{CDCl}_{3}$. (400 MHz, CDC/3), single peak at $\delta$ $-2.20$ 
675 Figure supplement 19 ESI-MS, m/z $838\left[\mathrm{M}^{+1}\right]^{+}$of POPC- $d_{77}$. Overall $93 \% \mathrm{D}$, isotope

676 distribution $d_{77}, 0 \%, d_{76}, 0 \%, d_{75}, 7.5 \%, d_{74}, 21.2 \%, d_{73}, 28.7 \%, d_{72}, 25.3 \%, d_{71}, 15.5 \%, d_{70}$,

$67710.8 \%$.

678 Figure supplement $20{ }^{1} \mathrm{H} N M R$ of choline- $d_{13}$ tetraphylborate (Figure 7, molecule 8) in

679 DMSO-d $d_{6}\left(400 \mathrm{MHz}, \mathrm{DMSO}-d_{6}\right) \delta 6.85(\mathrm{~m}, 4 \mathrm{H}), 6.96(\mathrm{~m}, 8 \mathrm{H}), 7.21(\mathrm{~m}, 8 \mathrm{H})$.

680 Figure supplement $21^{2} \mathrm{H}$ NMR of choline-d ${ }_{13}$ tetraphylborate (Figure 7, molecule 8) in 681 DMSO-d $.\left(61.4 \mathrm{MHz}, \mathrm{DMSO}-d_{6}\right) \delta 3.30(\mathrm{~m}, 9 \mathrm{D}), 3.32(\mathrm{~m}, 2 \mathrm{D}), 3.78(\mathrm{~m}, 2 \mathrm{D})$.

682 Figure supplement $22{ }^{13} \mathrm{C} \mathrm{NMR}$ of choline-d ${ }_{13}$ tetraphylborate (Figure 7, molecule 8) in 683 DMSO-d $.\left(100 \mathrm{MHz}, \mathrm{DMSO}-d_{6}\right) \delta 52.5(\mathrm{~m}), 54.9(\mathrm{~m}), 121.9,125.9,136.2,163.9(\mathrm{~m})$.

684 Figure supplement 23 POPC- $d_{77}$ percentage distribution of D levels at different sites.

685

686 CorA reconstitution in sND for SANS

687 CorA, d-csMSP1E3D1, and d-POPC solubilized in cholate were mixed in a ratio of 400:4:1 688 with a final d-POPC concentration of $10 \mathrm{mM}$. Cholate and DDM were removed by adding 50 $689 \% \mathrm{w} / \mathrm{v}$ amberlite XAD-2 overnight at $5^{\circ} \mathrm{C}$. CorA-loaded sNDs were purified by IMAC on NiNTA 690 resin, added TEV protease and dialysed for three hours at RT followed by ON dialysis at $4{ }^{\circ} \mathrm{C}$ against $20 \mathrm{mM}$ TrisHCl pH 8, $100 \mathrm{mM} \mathrm{NaCl}, 0.5 \mathrm{mM}$ EDTA, $1 \mathrm{mM}$ DTT. Finally, the cleaved sample was purified by reverse IMAC on NiNTA resin. The sample was concentrated to approximately $30 \mu \mathrm{M}$ before the SANS experiment.

694

\section{Reconstitution into multilamellar vesicles for NMR}

696

CorA was reconstituted into DMPC at a lipid-to-protein ratio of $0.5(\mathrm{w} / \mathrm{w})$ by dialysis against 10 $\mathrm{mM}$ Tris- $\mathrm{HCl} \mathrm{pH} 8,40 \mathrm{mM} \mathrm{MgCl} 2,1 \mathrm{mM}$ methyl- $\beta$-cyclodextrin using a $25 \mathrm{kDa} \mathrm{MWCO}$ membrane at RT. A white precipitate of multilamellar vesicles was visible after 48 hours and collected by centrifugation after 72 hours. Samples were packed into $1.3 \mathrm{~mm}$ or $0.7 \mathrm{~mm}$ MAS NMR rotors using an ultracentrifuge tool (Giotto Biotech) at $100.000 \mathrm{~g}$ for 1 hour.

701

702

\section{SEC-SANS}

703 Samples were measured at the D22 beamline at ILL, using the SEC-SANS mode described elsewhere ${ }^{26,27}$, but with the upgrade that UV absorption was measured on the flow cell in the same place, but perpendicular to the neutron beam. The setup was placed in a temperaturecontrolled cabinet at $11^{\circ} \mathrm{C}$. CorA in sDDM was run on a Superdex200 Increase 10/300 GL column (GE Healthcare) in $20 \mathrm{mM}$ TrisDCl pH 7.5, $150 \mathrm{mM} \mathrm{NaCl}, 1 \mathrm{mM}$ DTT, $0.5 \mathrm{mM}$ sDDM in $\mathrm{D}_{2} \mathrm{O}$ with an initial flow rate of $0.3 \mathrm{ml} / \mathrm{min}$ to allow full exchange into s-DDM ${ }^{29}$. CorA in sND was run on a Superose6 Increase 10/300 GL column (GE Healthcare) in $20 \mathrm{mM}$ TrisDCl pH 7.5, $150 \mathrm{mM} \mathrm{NaCl}, 1 \mathrm{mM}$ DTT in $\mathrm{D}_{2} \mathrm{O}$ with an initial flow rate of $0.5 \mathrm{ml} / \mathrm{min}$. For all samples, the flow was reduced to $0.05 \mathrm{ml} / \mathrm{min}$ during elution of the main peak. Measurements were run twice to obtain data from two sample-to-detector distances, $2 \mathrm{~m}$ and $11 \mathrm{~m}$, yielding data in a 
$713 q$-range of $0.0044 \AA^{-1}$ to $0.42 \AA^{-1}$ with the neutron wavelength of $6 \AA$. The scattering intensity,

$714 \quad I(q)$, was brought to absolute scale in units $\mathrm{fm}^{-1}$ by normalizing to the direct beam intensity

715 measured with an attenuator in place.

716

717 Pair distance, $p(r)$, distributions were calculated by Bayesian indirect Fourier transformation

718 at the bayesapp server ${ }^{32}$ (available from the genapp server at

$719 \mathrm{https}$ ://somo.chem.utk.edu/bayesapp/). $p(r)$ s and the corresponding $/(q)$ s for PDB structures

720 were calculated using CaPP (available at github.com/Niels-Bohr-Institute-XNS-

721 StructBiophys/CaPP) with a water layer excess density of $6 \%$ applied to the parts of the

722 protein outside the membrane.

723

\section{Model building}

725 For use in both SANS comparison, normal mode analysis, and simulations, we rebuilt the 726 missing residues and sidechains of the closed PDB structure 4I0U. The 2-6 missing residues 727 at each chain terminal was rebuilt using Modeller's automodel functionality ${ }^{49}$. In the open cryo728 EM structures, between $18-20$ residues were missing in the $\mathrm{N}$-terminal. These were rebuilt from the closed X-ray structure (4IOU) to allow for direct comparison to SANS measurements.

\section{Normal mode analysis}

732 Non-linear normal mode analysis was performed using the NOn-Linear rigid Block NMA ${ }^{50}$ 733 (NOLB) routine (available from https://hal.inria.fr/hal-01505843v2). The NOLB routine was 734 integrated with PEPSI-SANS 2.2 (https://team.inria.fr/nano-d/software/pepsi-sans/) and a 735 regularization algorithm to avoid unphysical structures. In the latter, $T=\chi^{2}+\alpha S$ is minimized, 736 where $S$ constraints the secondary structure deviation. Scanning over different values of $\alpha$, a 737 plot of $\chi^{2}$ vs. $S$ is obtained. The best compromise between conservation of structure and the 738 best fit to the data is chosen from the "elbow"-region (Figure 4-figure supplement 2).

\section{Metadynamics and reweighting}

741 The simulation system was set up using the MARTINI3.0b coarse grained force field ${ }^{51}$ with elastic networks terms applied to the individual chains only with a $0.9 \mathrm{~nm}$ cut-off and a force constant of $500 \mathrm{~kJ} /\left(\mathrm{mol} \mathrm{nm}{ }^{2}\right)$. As MARTINI does not contain parameters for protein bound $\mathrm{Mg}^{2+}$ but rather models it as a free ligand with four waters bound, the $\mathrm{Mg}^{2+}$ ions bound in the structure were deleted. To avoid overly electrostatic repulsions from the remaining $\mathrm{Mg}^{2+}$ coordinating amino acids (Asp89, Asp179, Asp253 and Glu88), they were transformed into their protonated states. The POPC membrane was obtained from CHARMM-GUI ${ }^{52,53}$ using 
conformational changes. The system was solvated with the MARTINI non-polarizable water and neutralized with $300 \mathrm{mM} \mathrm{NaCl}$ comparable to the SANS experimental set-up. The equilibration was performed according to the CHARMM-GUI equilibration protocol using a minimization step followed by 6 equilibration steps with slow decrease in the positional restraint forces on both lipids and protein in each step ${ }^{53}$.

The GROMACS-5.1.4 software package ${ }^{54}$ was used to simulate with a 20 fs time step. Temperature and semi-isotropic pressure were controlled at $303.15 \mathrm{~K}$ and 1 bar using the stochastic velocity rescaling thermostat ${ }^{55}$ and Parrinello-Rahman barostat ${ }^{56}$. Electrostatic interactions were modulated using the reaction field approach. The cutoff of short-range distance for the electrostatic interactions was $1.1 \mathrm{~nm}$. The potential shift Verlet scheme was used to cut off the Lennard-Jones potential at long ranges.

Well-Tempered Metadynamics ${ }^{57}$ simulations were performed with the PLUMED2.3.0 software $^{58}$. A radius of gyration collective variable was applied on all back- bone beads for the intracellular residues 170190 and 220250 of all 5 chains. The metadynamics parameters were set as follows: Gaussian width $=0.05$, Gaussian height $=2.1$, Gaussian de- position stride $=100$, biasfactor $=15$ and an upper wall defined at a CV radius of gyration of $4.0 \mathrm{~nm}$. The wall was defined as a harmonic restraint with a force constant $=10000$, harmonic exponential power $=4$, off-set $=0$, and a rescaling factor of 1 . Multiple wall types and sizes was attempted with lower walls causing too little dynamics for fitting with the experimental SANS data and higher walls causing individual monomers to bend unphysically and giving unfeasible large sample space. Clustering of the simulation trajectory was performed using the KMeans clustering method ${ }^{59}$.

The software program BME was used reweight the MetaD trajectory to fit the experimental SANS data ${ }^{60}$. The hyperparameter $\theta$ was determined based on a L-curve analysis (analogous to the procedure in normal mode analysis, see Figure 4-figure supplement 2) of $S_{\text {rel }}$ vs $\chi^{2}$, where $\theta$ is chosen where a natural kink is observed and any further decrease in $\chi^{2}$ gives rise to an increasing larger penalty in $S_{\text {rel. }}$. As the simulations are not fully converged and the chosen force field is coarse grained, we set the trust in the force field lower and chose a slightly lower $\theta$-value than the kink observed.

To account for a fixed fraction of symmetric pentamers, a differential intensity was derived by $I_{\text {diff }}=I_{\text {exp }}-f \cdot I_{\text {calc, 5sym, }}$ where $f$ is the fraction of symmetric pentamer, $I_{\exp }$ is the experimental SANS data, and $I_{\text {calc,5sym }}$ is the SANS signal of PDB 4IOU calculated by PEPSI-SANS. The 
785

786

787

788

789

790

791

792

793

794

795

796

797

798

799

800

801

802

803

804

805

806

807

808

809

810

811

812

813

814

815

816

817

818

819

820

821

forward scattering of the calculated SANS signal, $I_{\text {calc,5sym }}(0)$, was scaled to match the forward scattering of the experimental SANS data. Reweighting was done against the differential SANS signal.

\section{Solid-state NMR Spectroscopy}

Spectra of uniformly labelled samples were measured at a magnetic field of either $19.5 \mathrm{~T}$ or 23.4 T corresponding to a ${ }^{1} \mathrm{H}$ Larmor frequency of $800 \mathrm{MHz}$ and $1000 \mathrm{MHz}$, respectively. The spectrometers were equipped with a Bruker $0.7 \mathrm{~mm}$ MAS probe, spinning at $107 \mathrm{kHz}$, at a constant temperature of $300 \mathrm{~K}$. Spectra of ${ }^{15} \mathrm{~N}$ Ala labelled samples were acquired at $23.4 \mathrm{~T}$ on a Bruker $1.3 \mathrm{~mm}$ MAS probe, spinning at $60 \mathrm{kHz}$, at a constant temperature of $300 \mathrm{~K}$. The assignment of backbone resonances was preliminary obtained by acquiring a set of ${ }^{1} \mathrm{H}$ detected 3D experiments as described in ${ }^{61,62}$. Adamantane was used as the external reference. Spectral analysis and assignment were accomplished with CcpNmr Analysis ${ }^{63}$ and FLYA ${ }^{64}$.

Relaxation experiments were based on a ${ }^{1} \mathrm{H},{ }^{15} \mathrm{~N}{ }^{1} \mathrm{H}$-detected $\mathrm{CP}$-HSQC experiment incorporating an appropriate relaxation delay ${ }^{65} .38$ and 33 residues spanning different regions of the proteins were used for the uniformly labelled sample in the presence and in the absence of $\mathrm{Mg}^{2+}$, respectively. The measurements of site-specific ${ }^{15} \mathrm{~N} \mathrm{R}_{1 p}$ rates were performed at 107 $\mathrm{kHz}$ MAS, $19.5 \mathrm{~T}$ and $300 \mathrm{~K}$ and $280 \mathrm{~K}$, using relaxation delays of $0.05,1,5,15,50,100,200$ ms under a spin-lock field of $15 \mathrm{kHz}$. Measurements of bulk ${ }^{15} \mathrm{~N} \mathrm{R}_{1}$ were performed at 107 $\mathrm{kHz}$ MAS, $19.5 \mathrm{~T}$ and $300 \mathrm{~K}$, using relaxation delays of $0.5,1,2.8,6.8,15.8,23.8,53.5,80 \mathrm{~s}$. Measurements of ${ }^{15} \mathrm{~N} \mathrm{R}_{1 \rho}$ rates were additionally performed on the ${ }^{15} \mathrm{~N}$ Ala labelled samples in the presence and in the absence of $\mathrm{Mg}^{2+}$ at $60 \mathrm{kHz}$ MAS, $23.5 \mathrm{~T}$ and $300 \mathrm{~K}$ using relaxation delays of $0.1,1,5,10,25,50,100 \mathrm{~ms}$ under a spin-lock field of $15 \mathrm{kHz}$. The relaxation rates were obtained by fitting the experimental decay curves with a mono-exponential function. The error was estimated from the experimental noise by use of a Monte-Carlo evaluation.

\section{Activity assay}

Large unilamellar vesicles of POPC were prepared by dissolving a lipid film in $10 \mathrm{mM}$ MOPS$\mathrm{KOH} \mathrm{pH}$ 7.2, $150 \mathrm{mM} \mathrm{KCl}, 100 \mu \mathrm{M}$ EGTA including $10 \mu \mathrm{M}$ Mag-Fluo-4 (Thermo) to a POPC concentration of $15 \mathrm{mg} / \mathrm{ml}$, which was extruded through $0.2 \mu \mathrm{m}$ membrane filters for 35 times using a mini-extruder (Avanti Polar Lipids). CorA was inserted by mixing a sample of 10.5 mg/ml LUVs, $2 \mu \mathrm{M}$ CorA, $10 \mu \mathrm{M}$ Mag-Fluo-4 and 50 mM octyl glucoside. Biobeads SM-2 were added to $45 \% \mathrm{w} / \mathrm{v}$ and incubated at RT for $30 \mathrm{~min}$, before purifying and at the same time exchanging the extravesicular buffer to measurement buffer (10 mM MOPS-KOH pH 7.2, 150 $\mathrm{N}$-methyl-D-glucamine-HCl, $100 \mu \mathrm{M}$ EGTA) on Sephadex G50 resin. $20 \mu \mathrm{l}$ of CorA-LUVs (or plain LUVs) were diluted to a total of $1 \mathrm{ml}$ in measurement buffer (prepared in $\mathrm{H}_{2} \mathrm{O}$ or $\mathrm{D}_{2} \mathrm{O}$, 
822 respectively) containing $10 \mu \mathrm{M}$ valinomycin with or without $1 \mathrm{mM} \mathrm{Co}\left[\mathrm{NH}_{3}\right]^{3+}$ present. CorA

823 activity was monitored by Mag-Fluo-4 fluorescence at $515 \mathrm{~nm}$ (excitation at $488 \mathrm{~nm}$ ) on a

824 FluoroMax fluorometer (Horiba) upon addition of $10 \mathrm{mM} \mathrm{MgCl}_{2}$ (from a $1 \mathrm{M}$ stock prepared in

$825 \mathrm{H}_{2} \mathrm{O}$ or $\mathrm{D}_{2} \mathrm{O}$, respectively). The signal was normalized to the flat signal recorded before 826 addition of $\mathrm{MgCl}_{2}$.

827

828 Negative stain EM

829 CorA was purified by SEC and diluted to $0.1 \mu \mathrm{M}$ in appropriate buffers containing $1 \mathrm{mM}$ EDTA 830 or $40 \mathrm{mM} \mathrm{Mg}^{2+}$. Copper grids were neutralized with an Easiglow glow discharger (Agar 831 Scientific). $3 \mu$ of sample was applied to the grid and incubated for $30 \mathrm{~s}$. The grid was blotted 832 onto a filter paper from the edge, and $3 \mu \mathrm{L}$ of $2 \%$ uranyal formate was added immediately and 833 incubated for $30 \mathrm{~s}$. The staining procedure was repeated two more times. After the final 834 staining, the grid was left to dry for ten minutes. EM data were acquired on a Tecnai TEM (FEI, 835 Thermo Fischer scientific) at Aarhus University, Denmark. The micrographs were processed 836 by XMIPP to *.mcp files, and particle picking, 2D class averages and 3D model refinement 837 was done in Relion 3.0. Statistics for the 3D refinement are given in Table 1.

\begin{tabular}{|c|c|c|}
\hline Parameter & $1 \mathrm{mM}$ EDTA & $40 \mathrm{mM} \mathrm{MgCl} 2$ \\
\hline Pixel size, $\AA$ & 3.14 & 3.14 \\
\hline Number of micrographs & 436 & 440 \\
\hline Number of picked particles & 193606 & 185577 \\
\hline Final number of particles & 36176 & 46544 \\
\hline Resolution, $\AA$ & $\approx 15$ & $\approx 15$ \\
\hline
\end{tabular}

838 Table 1 Negative stain EM statistics for 3D model refinement.

\section{Acknowledgements}

840 We thank Elliot Gilbert for his assistance with SANS experiments at QUOKKA at ANSTO and

841 Marta Brennich for her assistance with SAXS experiments at BM29 at the ESRF. Thomas

842 Boesen is acknowledged for his help with EM experiments conducted at Aarhus University 843 and Michael Gajhede for his assistance in EM data processing. We thank Ida Louise 844 Jørgensen for helping with functional reconstitution of CorA in large unilamellar vesicles and 845 Michael Maguire for providing a plasmid encoding CorA. 


\section{Additional information}

847 Funding

\begin{tabular}{lll} 
Funder & Grant reference number & Author \\
\hline Lundbeck Foundation: Brainstruc & R155-2015-2666 & LA, KLL \\
\hline Novo Nordisk Foundation: Synergy & NNF15OC0016670 & LA \\
\hline $\begin{array}{l}\text { ERC: European Union's Horizon 2020 } \\
\text { research and innovation programme }\end{array}$ & ERC-2015-CoG GA 648974 & GP \\
\hline BBSRC & BB/R00126X/1; BB/N000145/1 & MS \\
\hline EPSRC & EP/R004722/1; & MS \\
& EP/R029407/1; EP/V010948/1 & \\
\hline Wellcome Trust & 208361/Z/17/Z & MS \\
\hline National Collaborative Research & & TD \\
Infrastructure Strategy & & \\
\hline European Commision iNext Discovery & GA 871037 & TS \\
\hline Villum Fonden & 35955 & NTJ
\end{tabular}

848

849 Competing interests

850 The authors declare no competing interests.

851

852 Author Contributions

853 LA, GP and KLL conceived the project. NTJ, JB, NY, and TS prepared samples. NTJ, AHL,

854 FGT, PH, AM, and MCP carried out SANS experiments and modeling. NTJ and TGP carried 855 out activity measurements. NTJ and AHL did EM experiments. TB, AHL, and RC carried out 856 simulations. MB, AB, and TS carried out NMR experiments and modeling. LA, GP, KLL, MR, 857 TGP, TD, and MS supervised the research. NTJ, MB, TS, LA, GP and KLL wrote the 858 manuscript with input from all authors.

859

\section{Data availability}

861 The SANS data have been deposited to the Small Angle Scattering Biological Data Bank 862 (https://www.sasbdb.org/, access codes SASDM42, SASDM52, SASDM62, SASDM72). The 863 NMR resonance assignments have been deposited in the Biological Magnetic Resonance 864 Data Bank (https://bmrb.io, access code 50959). The EM maps have been deposited to the 865 Electron Microscopy Data Bank (https://www.ebi.ac.uk/emdb, access codes EMD-13326 and 866 EMD-13327). Fluorescence data on CorA activity are available at GitHub https://github.com/Niels-Bohr-Institute-XNS-StructBiophys/CorADatal. The metadynamics 
simulations

are

available

at

GitHub

https://github.com/KULL-

869 Centre/papers/tree/main/2021/CorA-Johansen-et-al.

870

871

\section{Current affiliations}

872 NTJ: Section for Transport Biology, Department of Plant and Environmental Sciences,

873 University of Copenhagen, Frederiksberg, Denmark

874 AB: Institut des Sciences et Ingénierie Chimiques, École Polytechnique Fédérale de

875 Lausanne (EPFL), Lausanne, Switzerland;

876 RC: CSIC-Institute for Advanced Chemistry of Catalonia (IQAC), Barcelona, Catalonia,

877 Spain.

878

879

880

881

882

883

884

885

886

887

888

889

890

891

892

893

894

895

896

897

898

899

900

901

902

903

904

905

906

907

908

909

\section{References}

1. Jahnen-Dechent, W. \& Ketteler, M. Magnesium basics. Clinical Kidney Journal 5, i3i14 (2012).

2. Rude, R. K. Magnesium Deficiency: A Cause of Heterogenous Disease in Humans. Journal of Bone and Mineral Research 13, 749-758 (1998).

3. de Baaij, J. H. F., Hoenderop, J. G. J. \& Bindels, R. J. M. Magnesium in man: Implications for health and disease. Physiological Reviews 95, 1-46 (2015).

4. Dinicolantonio, J. J., Liu, J. \& O'Keefe, J. H. Magnesium for the prevention and treatment of cardiovascular disease. Open Heart 5, 1-10 (2018).

5. Maguire, M. E. Magnesium transporters: properties, regulation and structure. Frontiers in bioscience : a journal and virtual library 11, 3149-63 (2006).

6. Papp-Wallace, K. M. \& Maguire, M. E. Bacterial homologs of eukaryotic membrane proteins: The 2-TM-GxN family of Mg2+ transporters (Review). Molecular Membrane

Biology 24, 351-356 (2007).

7. Knoop, V., Groth-Malonek, M., Gebert, M., Eifler, K. \& Weyand, K. Transport of magnesium and other divalent cations: Evolution of the 2-TM-GxN proteins in the MIT superfamily. Molecular Genetics and Genomics 274, 205-216 (2005).

8. Lunin, V. v et al. Crystal structure of the CorA Mg2+ transporter. Nature 440, 833-837 (2006).

9. Eshaghi, S. Crystal Structure of a Divalent Metal Ion Transporter CorA at 2.9 Angstrom Resolution. Science 313, 354-357 (2006).

10. Payandeh, J. \& Pai, E. F. A structural basis for Mg2+ homeostasis and the CorA translocation cycle. EMBO Journal 25, 3762-3773 (2006).

11. Nordin, N. et al. Exploring the structure and function of Thermotoga maritima CorA reveals the mechanism of gating and ion selectivity in Co $2+/ \mathrm{Mg} 2+$ transport. Biochemical Journal 451, 365-374 (2013).

12. Pfoh, R. et al. Structural asymmetry in the magnesium channel CorA points to sequential allosteric regulation. Proceedings of the National Academy of Sciences 109, 18809-18814 (2012).

13. Palombo, I., Daley, D. O. \& Rapp, M. Why Is the GMN Motif Conserved in the CorA/Mrs2/Alr1 Superfamily of Magnesium Transport Proteins? Biochemistry 52, 4842-4847 (2013). 
14. Dalmas, O. et al. A repulsion mechanism explains magnesium permeation and selectivity in CorA. Proceedings of the National Academy of Sciences 111, 30023007 (2014).

15. Payandeh, J. et al. Probing Structure-Function Relationships and Gating Mechanisms in the CorA Mg 2+ Transport System. Journal of Biological Chemistry 283, 1172111733 (2008).

16. Dalmas, O., Sompornpisut, P., Bezanilla, F. \& Perozo, E. Molecular mechanism of Mg2+-dependent gating in CorA. Nature Communications 5, 3590 (2014).

17. Chakrabarti, N., Neale, C., Payandeh, J., Pai, E. F. \& Pomès, R. An iris-like mechanism of pore dilation in the CorA magnesium transport system. Biophysical Journal 98, 784-792 (2010).

18. Guskov, A. et al. Structural insights into the mechanisms of Mg2+ uptake, transport, and gating by CorA. Proceedings of the National Academy of Sciences 109, 1845918464 (2012).

19. Kowatz, T. \& Maguire, M. E. BBA - General Subjects Loss of cytosolic Mg $2+$ binding sites in the Thermotoga maritima CorA Mg $2+$ channel is not su ffi cient for channel opening. BBA - General Subjects 1863, 25-30 (2019).

20. Matthies, D. et al. Cryo-EM Structures of the Magnesium Channel CorA Reveal Symmetry Break upon Gating. Cell 164, 747-756 (2016).

21. Neale, C., Chakrabarti, N., Pomorski, P., Pai, E. F. \& Pomès, R. Hydrophobic Gating of Ion Permeation in Magnesium Channel CorA. PLOS Computational Biology 11, e1004303 (2015).

22. Nemchinova, M., Melcr, J., Wassenaar, T. A., Marrink, S. J. \& Guskov, A. Asymmetric CorA Gating Mechanism as Observed by Molecular Dynamics Simulations. Journal of Chemical Information and Modeling acs.jcim.1c00261 (2021) doi:10.1021/acs.jcim.1c00261.

23. Rangl, M., Schmandt, N., Perozo, E. \& Scheuring, S. Real time dynamics of gatingrelated conformational changes in CorA. eLife 8, 1-17 (2019).

24. Reif, B., Ashbrook, S. E., Emsley, L. \& Hong, M. Solid-state NMR spectroscopy. Nature Reviews Methods Primers 1, 2 (2021).

25. Bonaccorsi, M., le Marchand, T. \& Pintacuda, G. Protein structural dynamics by Magic-Angle Spinning NMR. Current Opinion in Structural Biology 70, 34-43 (2021).

26. Johansen, N. T., Pedersen, M. C., Porcar, L., Martel, A. \& Arleth, L. Introducing SECSANS for studies of complex self-organized biological systems. Acta Crystallographica Section D Structural Biology 74, 1178-1191 (2018).

27. Jordan, A. et al. SEC-SANS: size exclusion chromatography combined in situ with small-angle neutron scattering. Journal of Applied Crystallography 49, 2015-2020 (2016).

28. Maric, S. et al. Stealth carriers for low-resolution structure determination of membrane proteins in solution. Acta Crystallographica Section D Biological Crystallography 70, 317-328 (2014).

29. Midtgaard, S. R. et al. Invisible detergents for structure determination of membrane proteins by small-angle neutron scattering. FEBS Journal 285, 357-371 (2018).

30. Schubeis, T., le Marchand, T., Andreas, L. B. \& Pintacuda, G. 1H magic-angle spinning NMR evolves as a powerful new tool for membrane proteins. Journal of Magnetic Resonance 287, 140-152 (2018). 
31. Schubeis, T. et al. A $\beta$-barrel for oil transport through lipid membranes: Dynamic NMR structures of AlkL. Proceedings of the National Academy of Sciences of the United States of America 117, 21014-21021 (2020).

32. Hansen, S. Update for BayesApp : a web site for analysis of small-angle scattering data. Journal of Applied Crystallography 47, 1469-1471 (2014).

33. Skar-Gislinge, N. et al. Elliptical Structure of Phospholipid Bilayer Nanodiscs Encapsulated by Scaffold Proteins: Casting the Roles of the Lipids and the Protein. Journal of the American Chemical Society 132, 13713-13722 (2010).

34. Pedersen, M. C., Arleth, L. \& Mortensen, K. WillttFit : a framework for fitting of constrained models to small-angle scattering data. Journal of Applied Crystallography 46, 1894-1898 (2013).

35. Némethy, G. \& Scheraga, H. A. Structure of Water and Hydrophobic Bonding in Proteins. IV. The Thermodynamic Properties of Liquid Deuterium Oxide. The Journal of Chemical Physics 41, 680-689 (1964).

36. Sugiyama, T. \& Yoshiok, T. Functional Difference Between Deuterated and Protonated Macromolecules. in Protein Structure (ed. Faraggi, E.) 291-308 (InTech, 2012). doi:10.5772/36649.

37. Hummer, G., Garde, S., García, A. E. \& Pratt, L. R. New perspectives on hydrophobic effects. Chemical Physics 258, 349-370 (2000).

38. Franke, D. \& Svergun, D. I. DAMMIF, a program for rapid ab-initio shape determination in small-angle scattering. Journal of Applied Crystallography 42, 342346 (2009).

39. Franke, D. et al. ATSAS 2.8: A comprehensive data analysis suite for small-angle scattering from macromolecular solutions. Journal of Applied Crystallography 50, 1212-1225 (2017).

40. Payandeh, J. \& Pai, E. F. A structural basis for Mg2+ homeostasis and the CorA translocation cycle. The EMBO Journal 25, 3762-3773 (2006).

41. Chakrabarti, N., Neale, C., Payandeh, J., Pai, E. F. \& Pomès, R. An iris-like mechanism of pore dilation in the CorA magnesium transport system. Biophysical Journal 98, 784-792 (2010).

42. Gati, C., Stetsenko, A., Slotboom, D. J., Scheres, S. H. W. \& Guskov, A. The structural basis of proton driven zinc transport by ZntB. Nature Communications $\mathbf{8}$, 1313 (2017).

43. Hu, J., Sharma, M., Qin, H., Gao, F. P. \& Cross, T. A. Ligand Binding in the Conserved Interhelical Loop of CorA, a Magnesium Transporter from Mycobacterium tuberculosis. Journal of Biological Chemistry 284, 15619-15628 (2009).

44. Rao, S., Klesse, G., Lynch, C. I., Tucker, S. J. \& Sansom, M. S. P. Molecular Simulations of Hydrophobic Gating of Pentameric Ligand Gated Ion Channels: Insights into Water and lons. Journal of Physical Chemistry B 125, 981-994 (2021).

45. Zhang, Y. et al. Asymmetric opening of the homopentameric 5-HT3A serotonin receptor in lipid bilayers. Nature Communications 12, 1074 (2021).

46. Unwin, N. \& Fujiyoshi, Y. Gating Movement of Acetylcholine Receptor Caught by Plunge-Freezing. Journal of Molecular Biology 422, 617-634 (2012).

47. Yepuri, N. R. et al. Synthesis of Perdeuterated 1-Palmitoyl-2-oleoyl- sn -glycero-3phosphocholine ([D 82 ]POPC) and Characterisation of Its Lipid Bilayer Membrane Structure by Neutron Reflectometry. ChemPlusChem 81, 315-321 (2016). 
48. Johansen, N. T. et al. Circularized and solubility-enhanced MSPs facilitate simple and high yield production of stable nanodiscs for studies of membrane proteins in solution. The FEBS Journal 0, 1734-1751 (2019).

49. Webb, B. \& Sali, A. Protein Structure Modeling with MODELLER. in Functional Genomics: Methods and Protocols (eds. Kaufmann, M., Klinger, C. \& Savelsbergh, A.) 39-54 (Springer New York, 2017). doi:10.1007/978-1-4939-7231-9_4.

50. Hoffmann, A. \& Grudinin, S. NOLB: Nonlinear Rigid Block Normal-Mode Analysis Method. Journal of Chemical Theory and Computation 13, 2123-2134 (2017).

51. Marrink, S. J. Martini 3 open-beta release. http://cgmartini.nl/index.php/martini3beta.

52. Jo, S., Lim, J. B., Klauda, J. B. \& Im, W. CHARMM-GUI Membrane Builder for Mixed Bilayers and Its Application to Yeast Membranes. Biophysical Journal 97, 50-58 (2009).

53. Qi, Y. et al. CHARMM-GUI Martini Maker for Coarse-Grained Simulations with the Martini Force Field. Journal of Chemical Theory and Computation 11, 4486-4494 (2015).

54. Abraham, M. J. et al. GROMACS: High performance molecular simulations through multi-level parallelism from laptops to supercomputers. SoftwareX 1-2, 19-25 (2015).

55. Bussi, G., Donadio, D. \& Parrinello, M. Canonical sampling through velocity rescaling. The Journal of Chemical Physics 126, 014101 (2007).

56. Parrinello, M. \& Rahman, A. Polymorphic transitions in single crystals: A new molecular dynamics method. Journal of Applied Physics 52, 7182-7190 (1981).

57. Barducci, A., Bussi, G. \& Parrinello, M. Well-Tempered Metadynamics: A Smoothly Converging and Tunable Free-Energy Method. Physical Review Letters 100, 020603 (2008).

58. Tribello, G. A., Bonomi, M., Branduardi, D., Camilloni, C. \& Bussi, G. PLUMED 2: New feathers for an old bird. Computer Physics Communications 185, 604-613 (2014).

59. Tiberti, M., Papaleo, E., Bengtsen, T., Boomsma, W. \& Lindorff-Larsen, K. ENCORE: Software for Quantitative Ensemble Comparison. PLoS Computational Biology 11, 116 (2015).

60. Bottaro, S., Bengtsen, T. \& Lindorff-Larsen, K. Integrating Molecular Simulation and Experimental Data: A Bayesian/Maximum Entropy Reweighting Approach. in Structural Bioinformatics: Methods and Protocols (ed. Gáspári, Z.) 219-240 (Springer US, 2020). doi:10.1007/978-1-0716-0270-6_15.

61. Barbet-Massin, E. et al. Rapid Proton-Detected NMR Assignment for Proteins with Fast Magic Angle Spinning. Journal of the American Chemical Society 136, 1248912497 (2014).

62. Stanek, J. et al. NMR Spectroscopic Assignment of Backbone and Side-Chain Protons in Fully Protonated Proteins: Microcrystals, Sedimented Assemblies, and Amyloid Fibrils. Angewandte Chemie International Edition 55, 15504-15509 (2016).

63. Vranken, W. F. et al. The CCPN data model for NMR spectroscopy: Development of a software pipeline. Proteins: Structure, Function and Genetics 59, 687-696 (2005).

64. Schmidt, E. \& Güntert, P. A new algorithm for reliable and general NMR resonance assignment. Journal of the American Chemical Society 134, 12817-12829 (2012).

65. Knight, M. J. et al. Structure and backbone dynamics of a microcrystalline metalloprotein by solid-state NMR. Proceedings of the National Academy of Sciences of the United States of America 109, 11095-11100 (2012). 
A

mone B

$410 U$

3JCG

GMN filter-

TMD

ICD
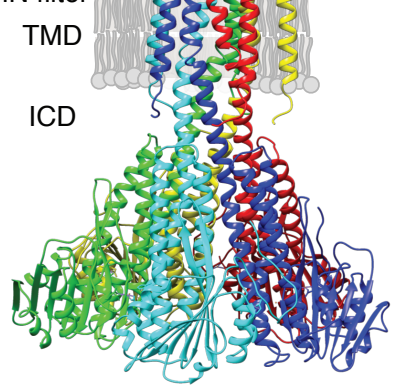

का 1 की है।

क 30 क

2.

knes

त. है करल $8 \leq 26$

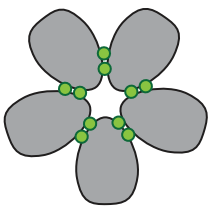

हैं?

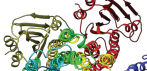

8 s sesmo

89 onv

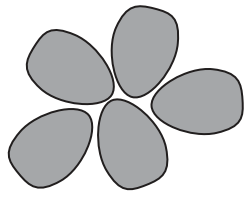



$A$

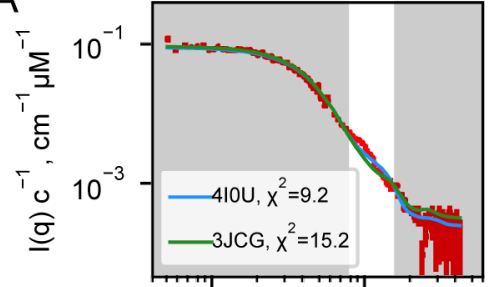

$\begin{array}{lr}10 \\ 0 \\ \overline{4} & -10\end{array}$

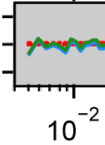

q. $\AA^{-1}$

C

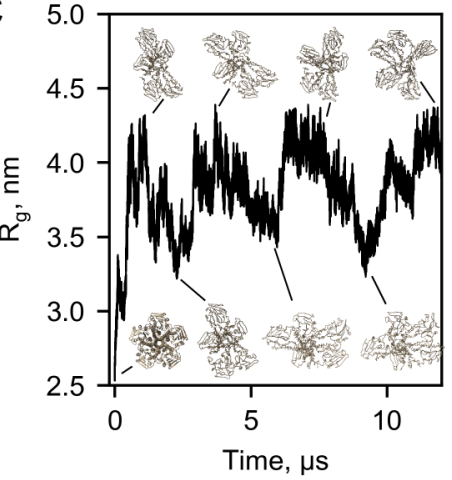

B

$T$

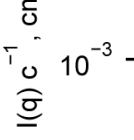

\begin{tabular}{rr}
0 & 10 \\
\hdashline & 0 \\
\hline$\triangleleft$ & -10
\end{tabular}

$10^{-2}$

$10^{-1}$

q. $\AA^{-1}$

D

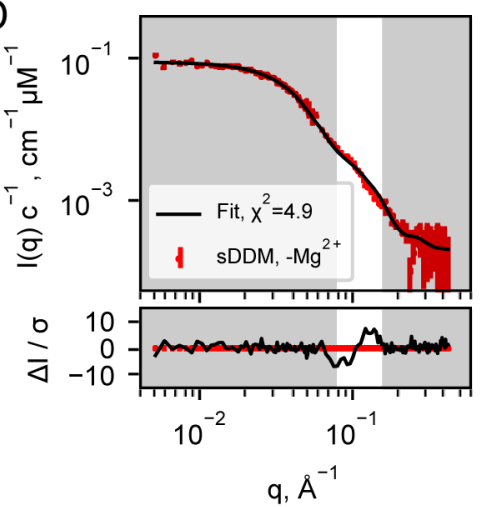

E

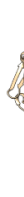

s.

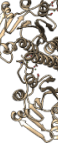

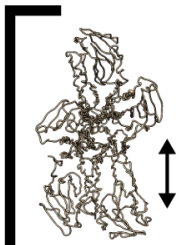

$45.9 \%$

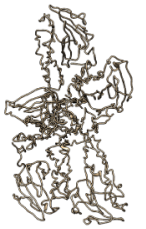

$3.2 \%$

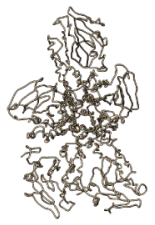

$2.8 \%$

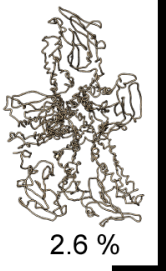

Input

BME clusters $(w>2.5 \%)$ 
$\mathrm{A}^{+\mathrm{Mg}^{2+}}-\mathrm{Mg}^{2+}$

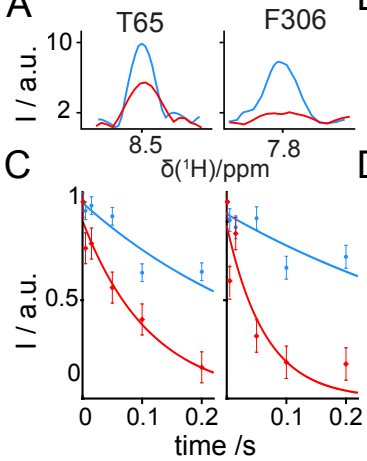

B

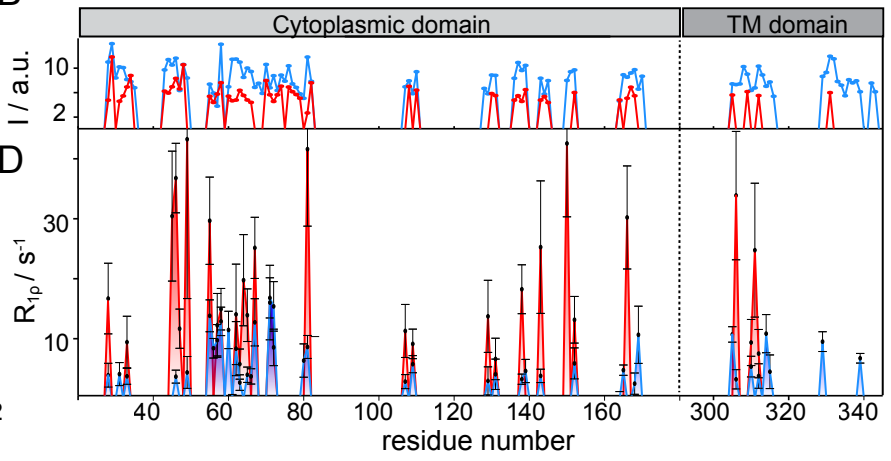




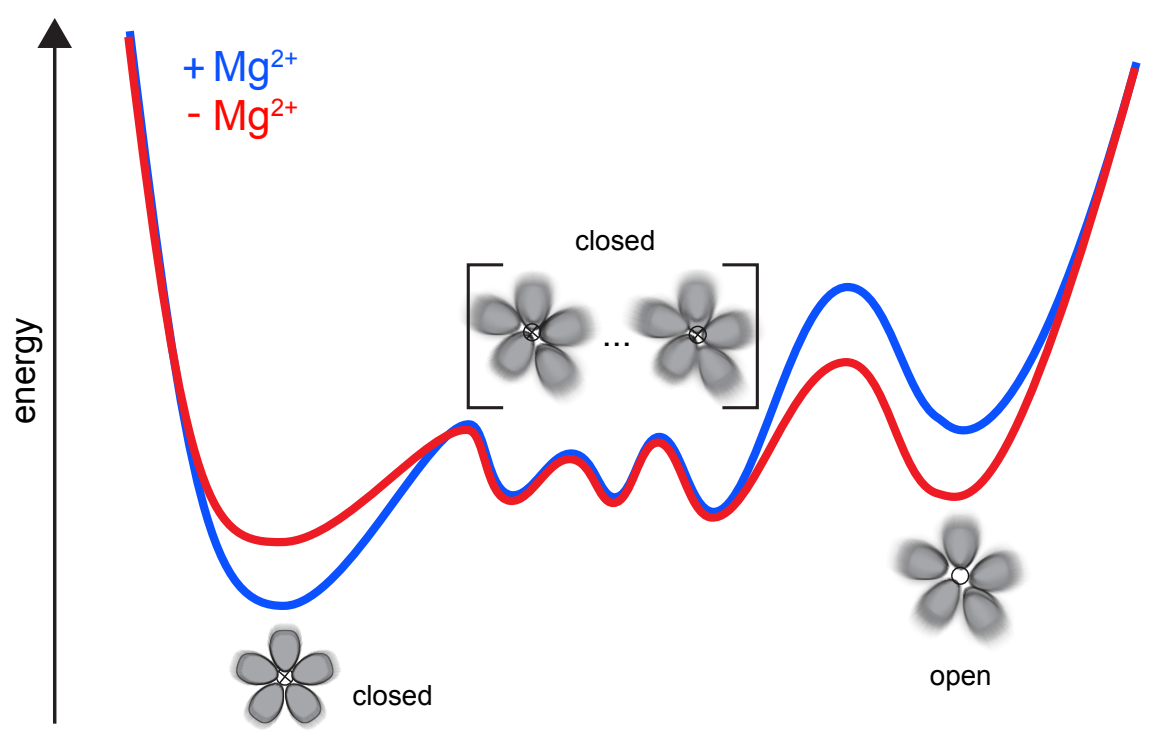



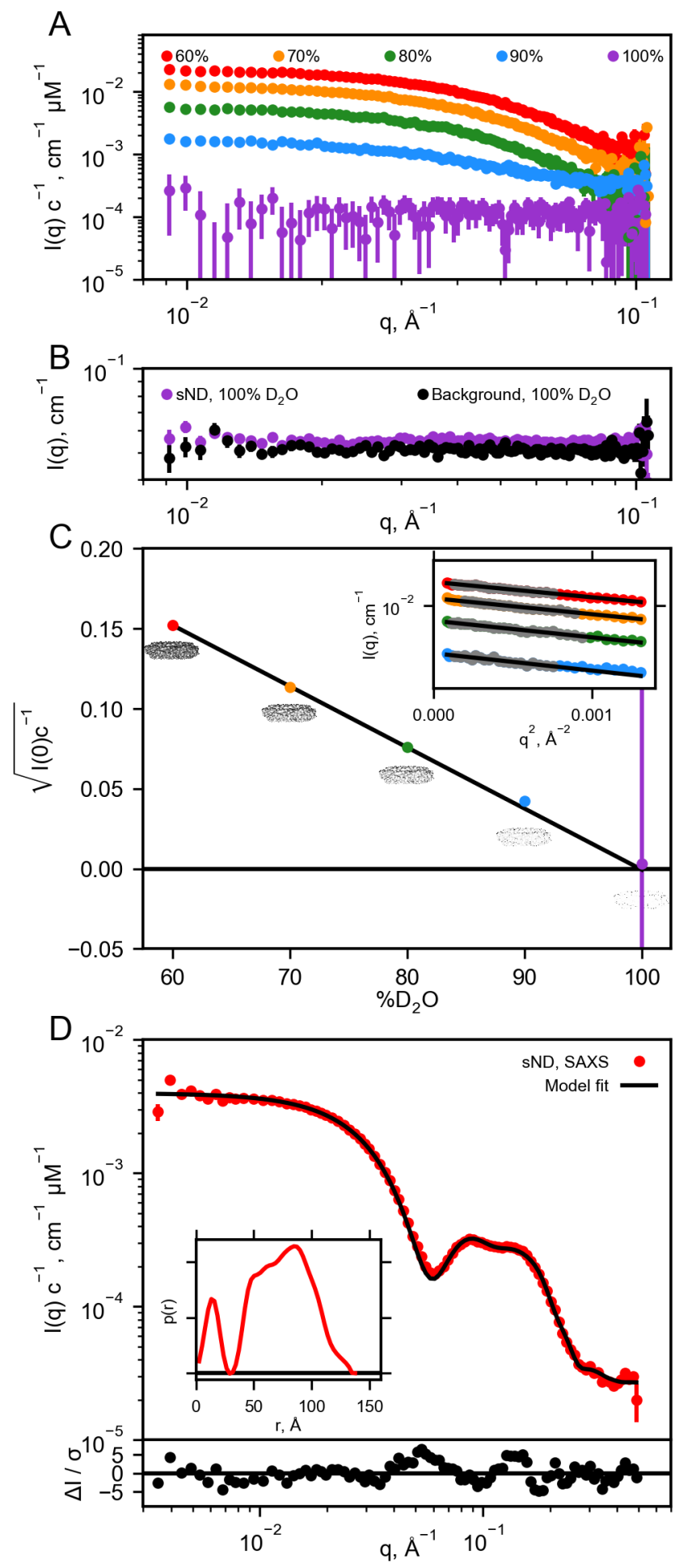

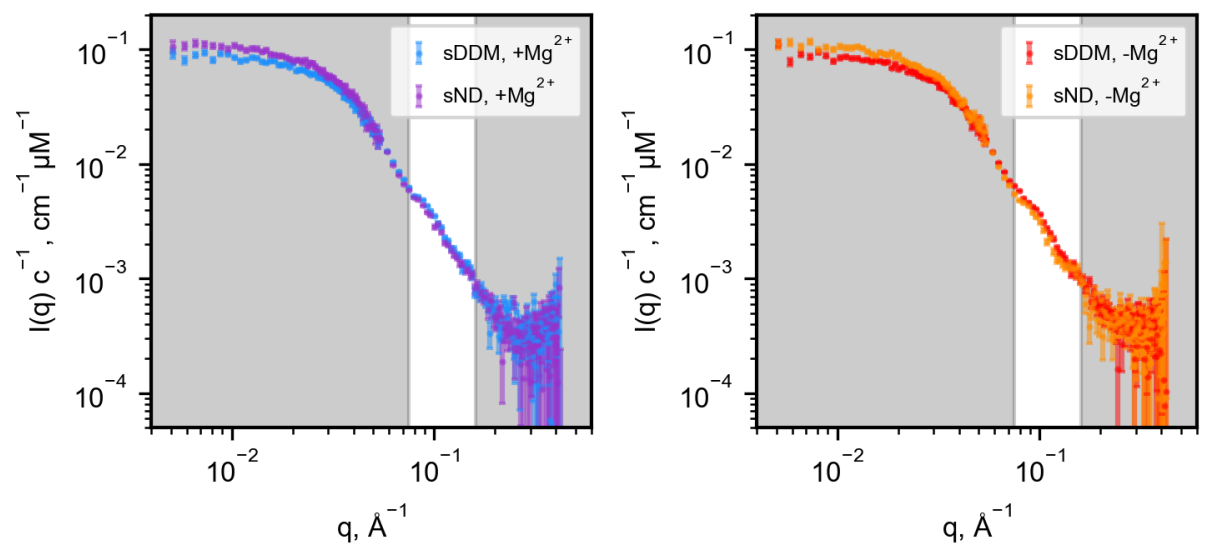

q. $\AA^{-1}$ 
in SDDM, w/o $\mathrm{Mg}^{2+}$
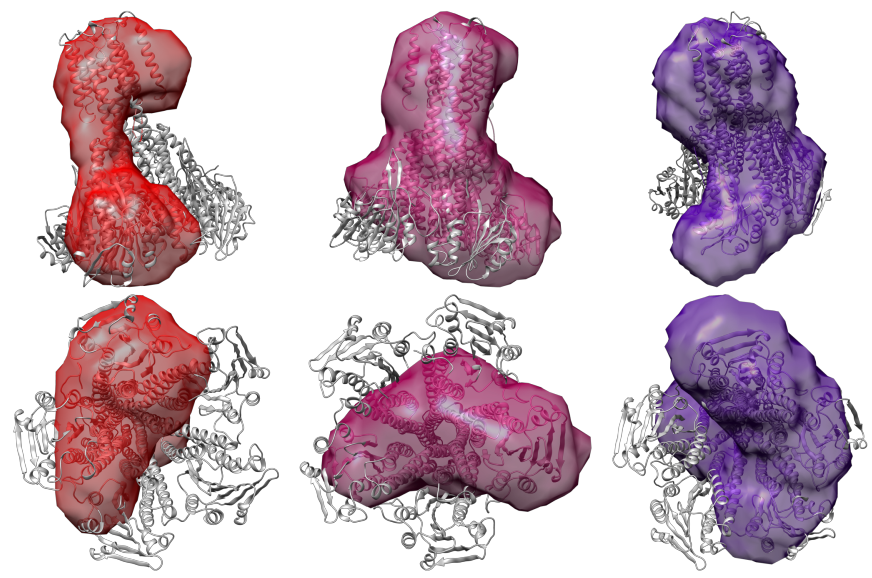

P5 symmetry imposed
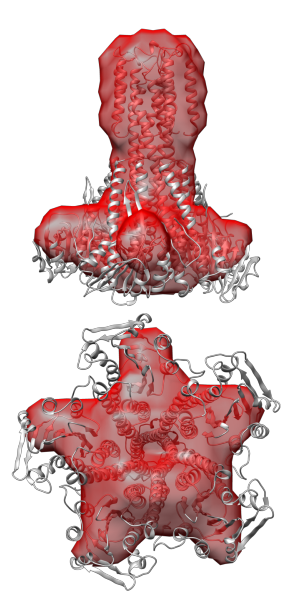

in SDDM, w/o $\mathrm{Mg}^{2+}$ in $\mathrm{sDDM}, \mathrm{w} / \mathrm{Mg}^{2+}$

in $\mathrm{SND}, \mathrm{w} / \mathrm{O} \mathrm{Mg}^{2+}$

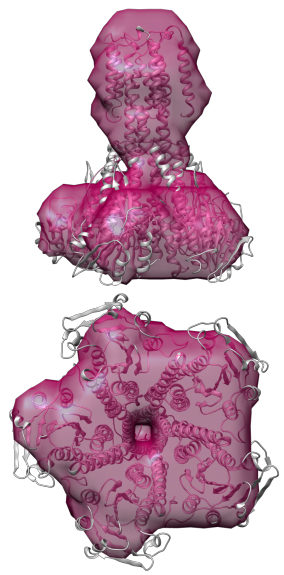

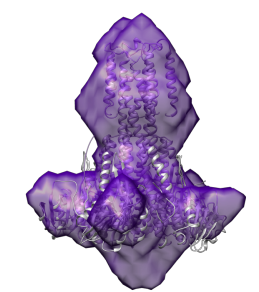

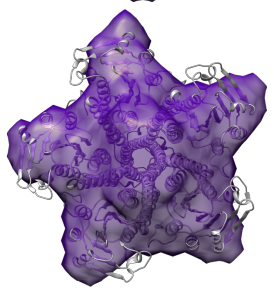

in $\mathrm{SND}, \mathrm{w} / \mathrm{Mg}^{2+}$

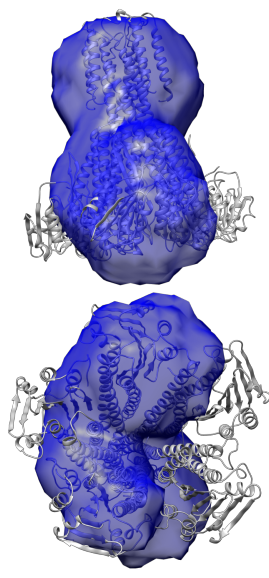




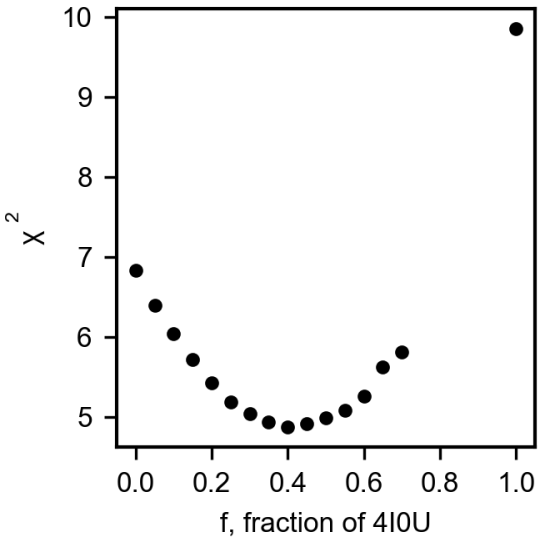




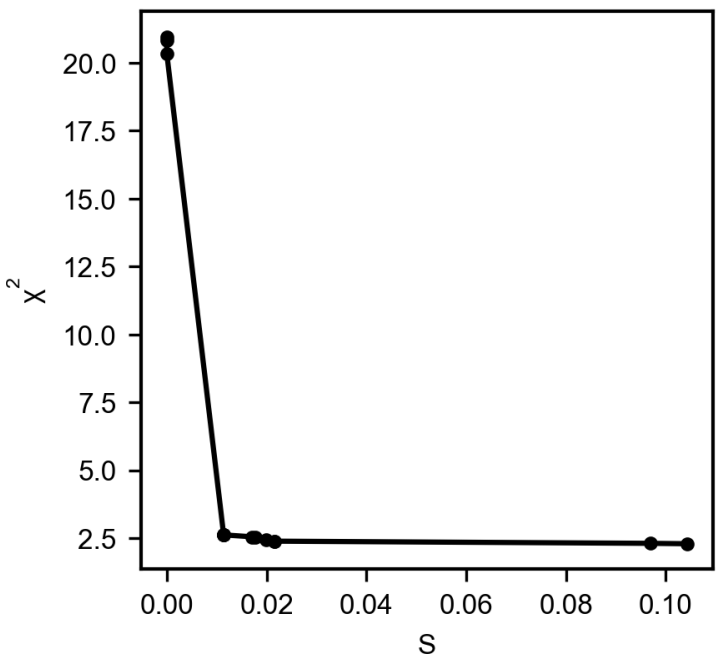




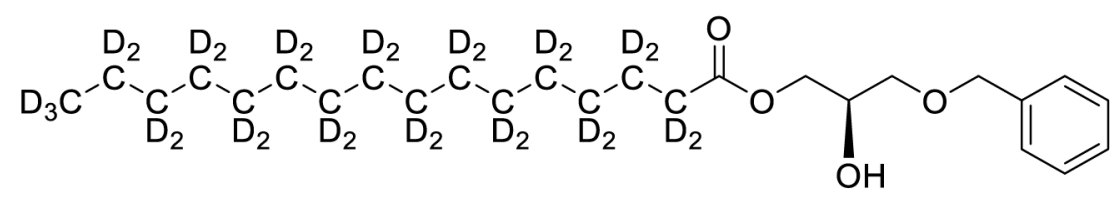




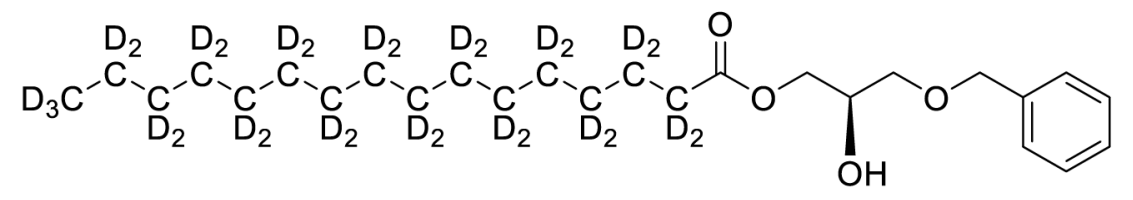

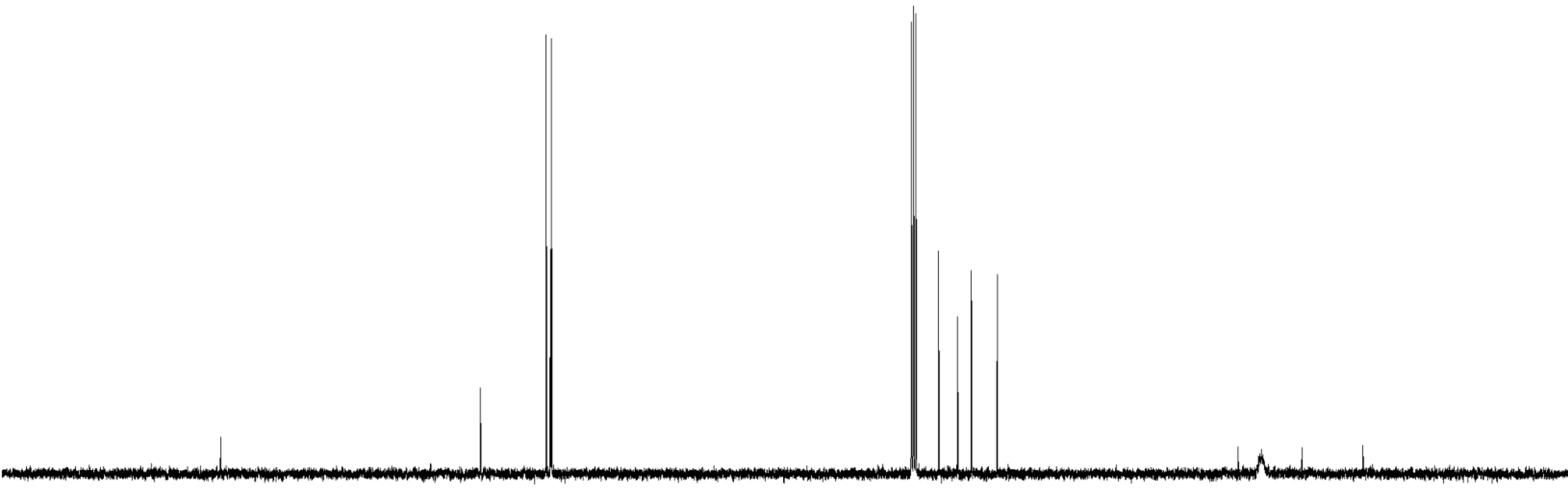

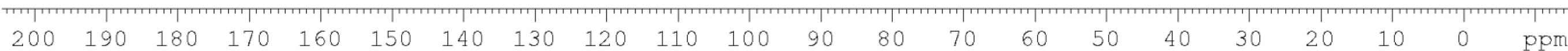



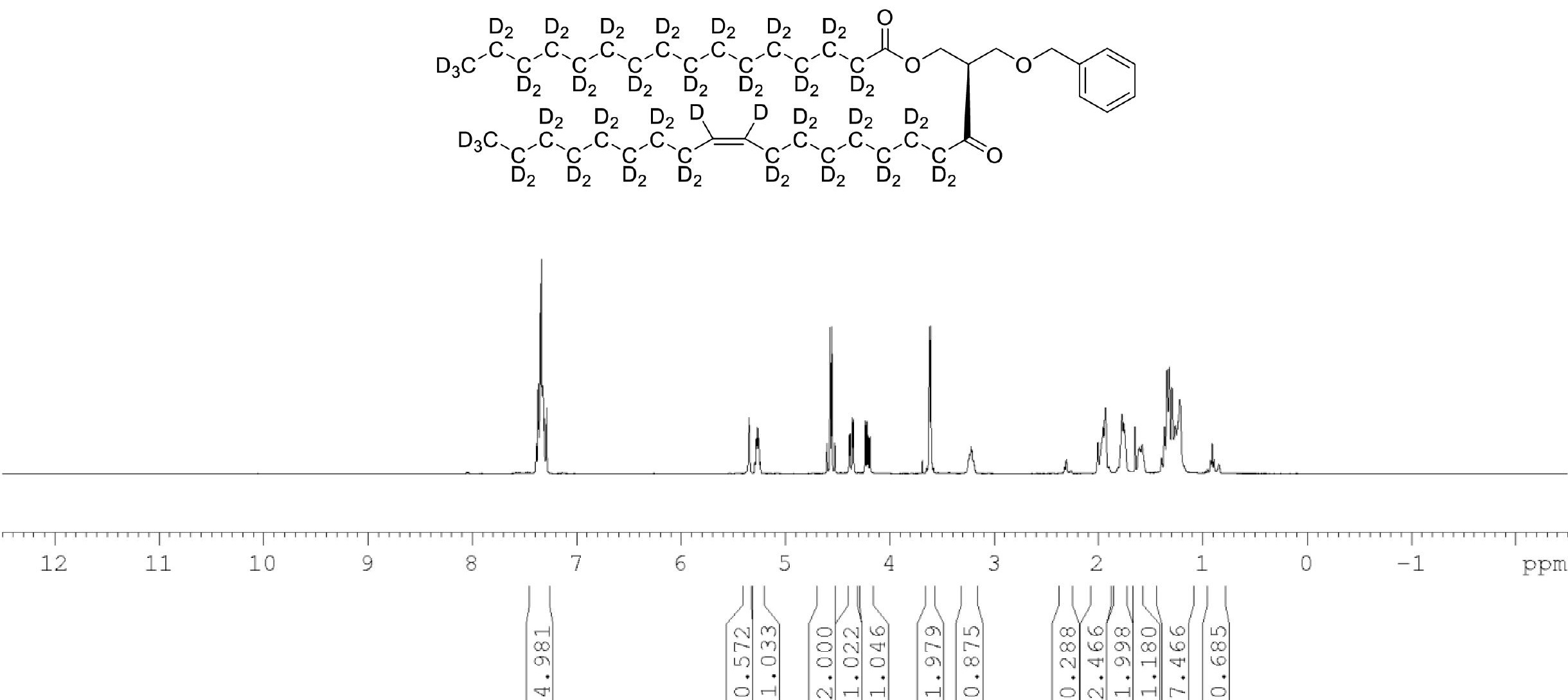


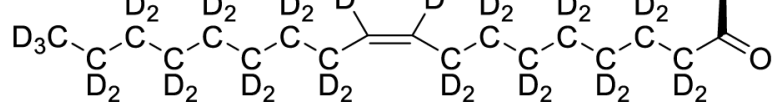




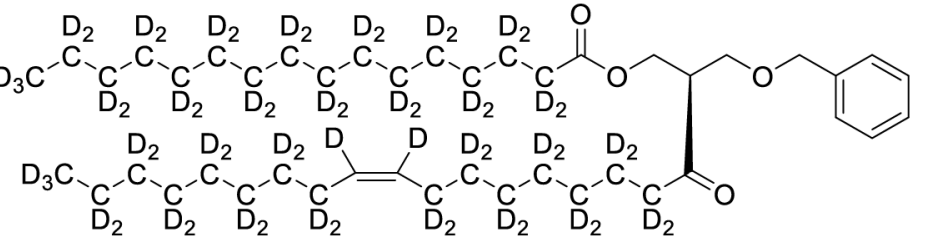

Solvent EtOAc traces

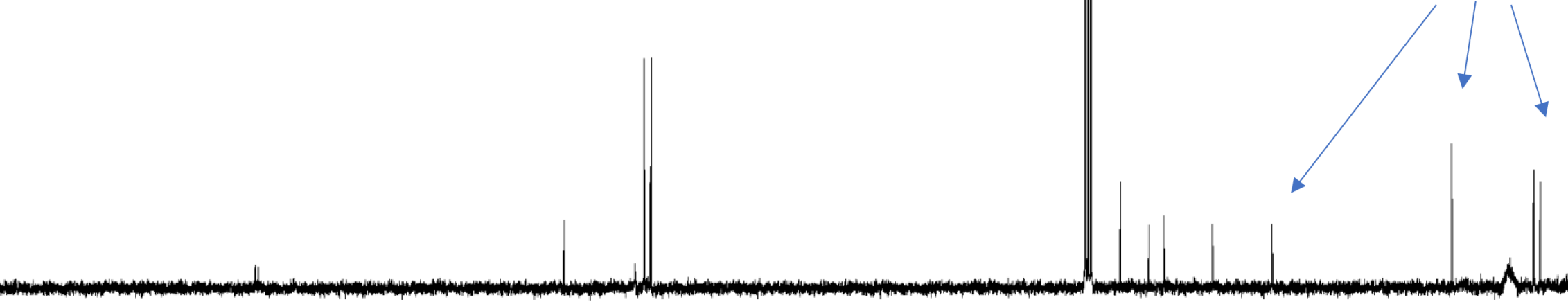




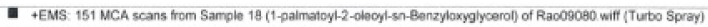

$19 \mathrm{e} 7$

$1 \mathrm{Be} 7$

1.7e?

$16 \mathrm{e} 7$

1.507

14 e?

1.3e?

$12 \mathrm{e} 7$

1.1e?

굴 $10 \mathrm{et}$

8.0e6

E 8006

70 es

$60 \mathrm{e} 6$

50 es

40 es

$30 \mathrm{e} 6$

20 es

$1.0 \mathrm{e} 6$ $1.0 \mathrm{e} 6731.4 \quad 737.7381$ 730

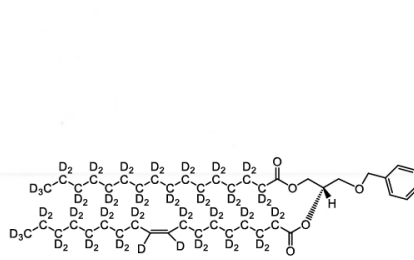

7640

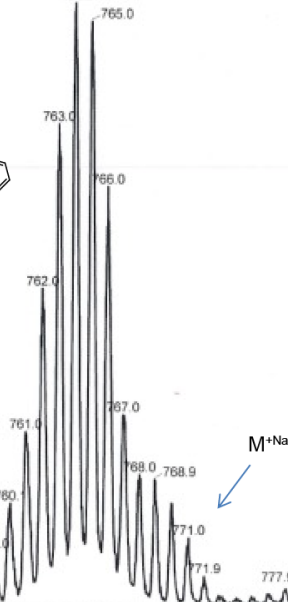

$\mathrm{M}^{+\mathrm{Na}}$ 


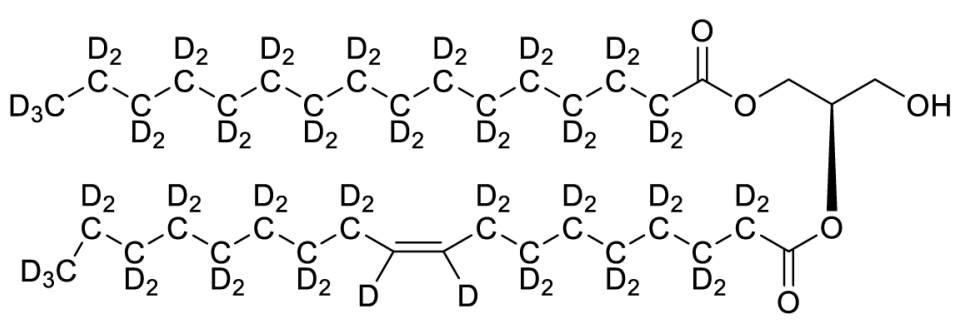




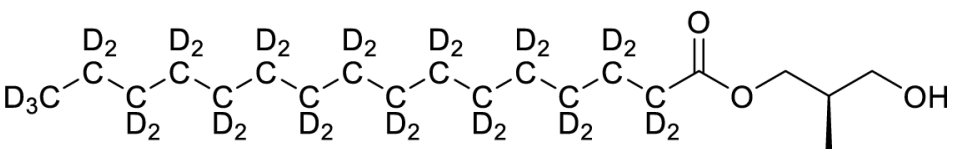

$$
\begin{aligned}
& \begin{array}{llllllll}
\mathrm{D}_{2} & \mathrm{D}_{2} & \mathrm{D}_{2} & \mathrm{D}_{2} & \mathrm{D}_{2} & \mathrm{D}_{2} & \mathrm{D}_{2} & \mathrm{D}_{2}
\end{array}
\end{aligned}
$$

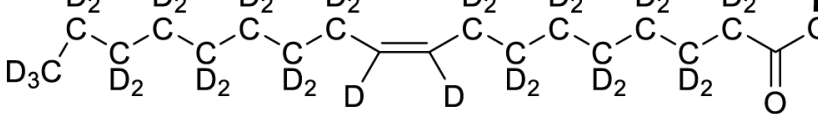



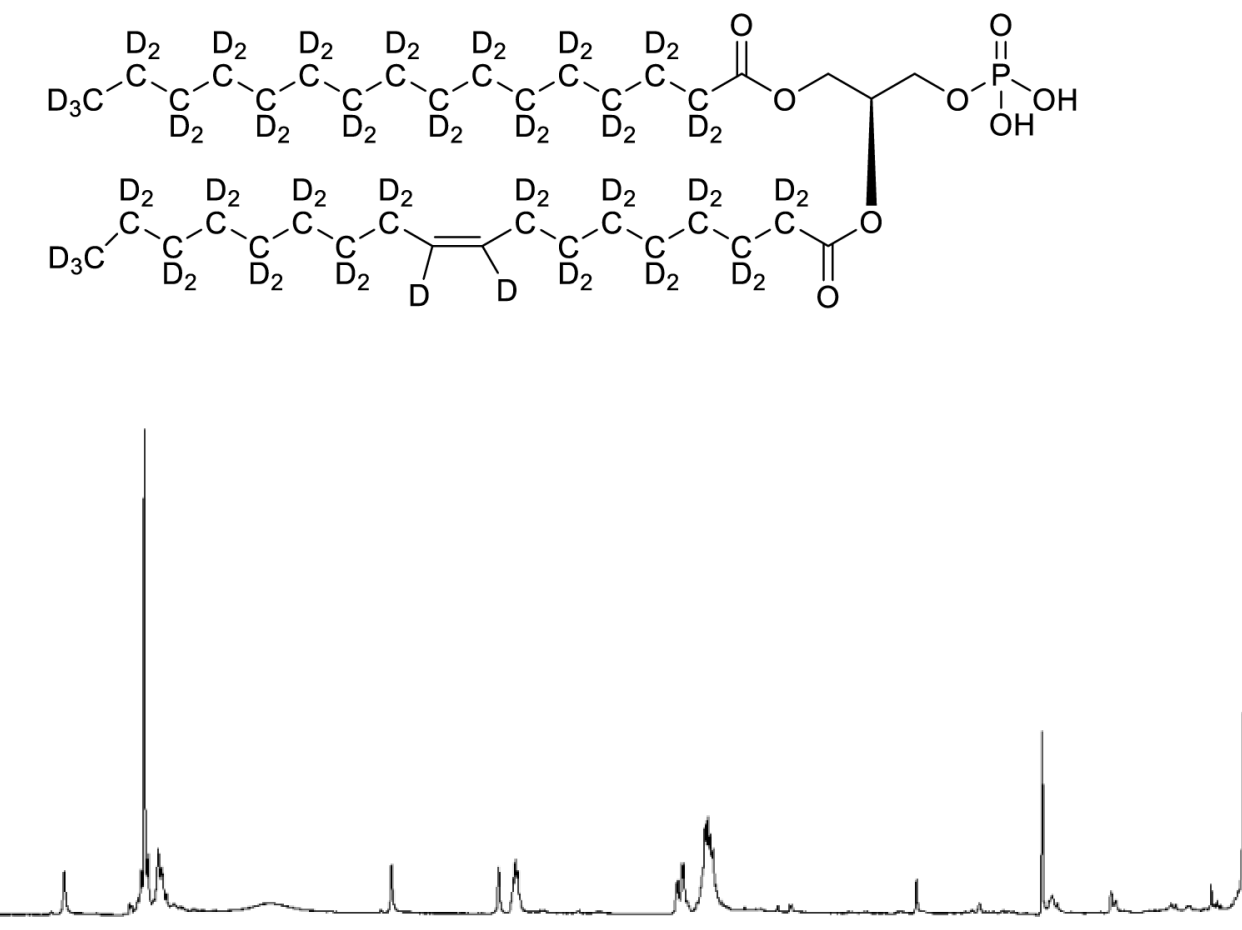


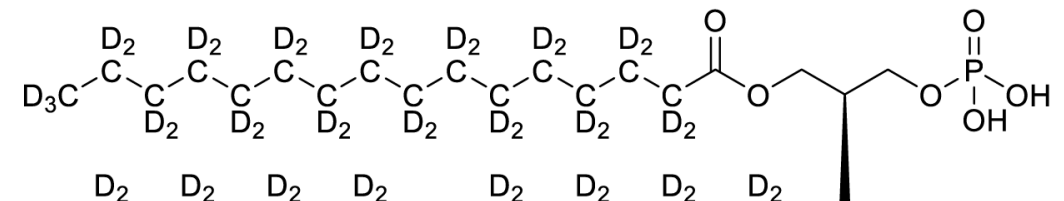

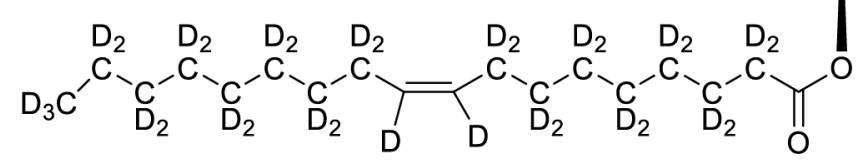




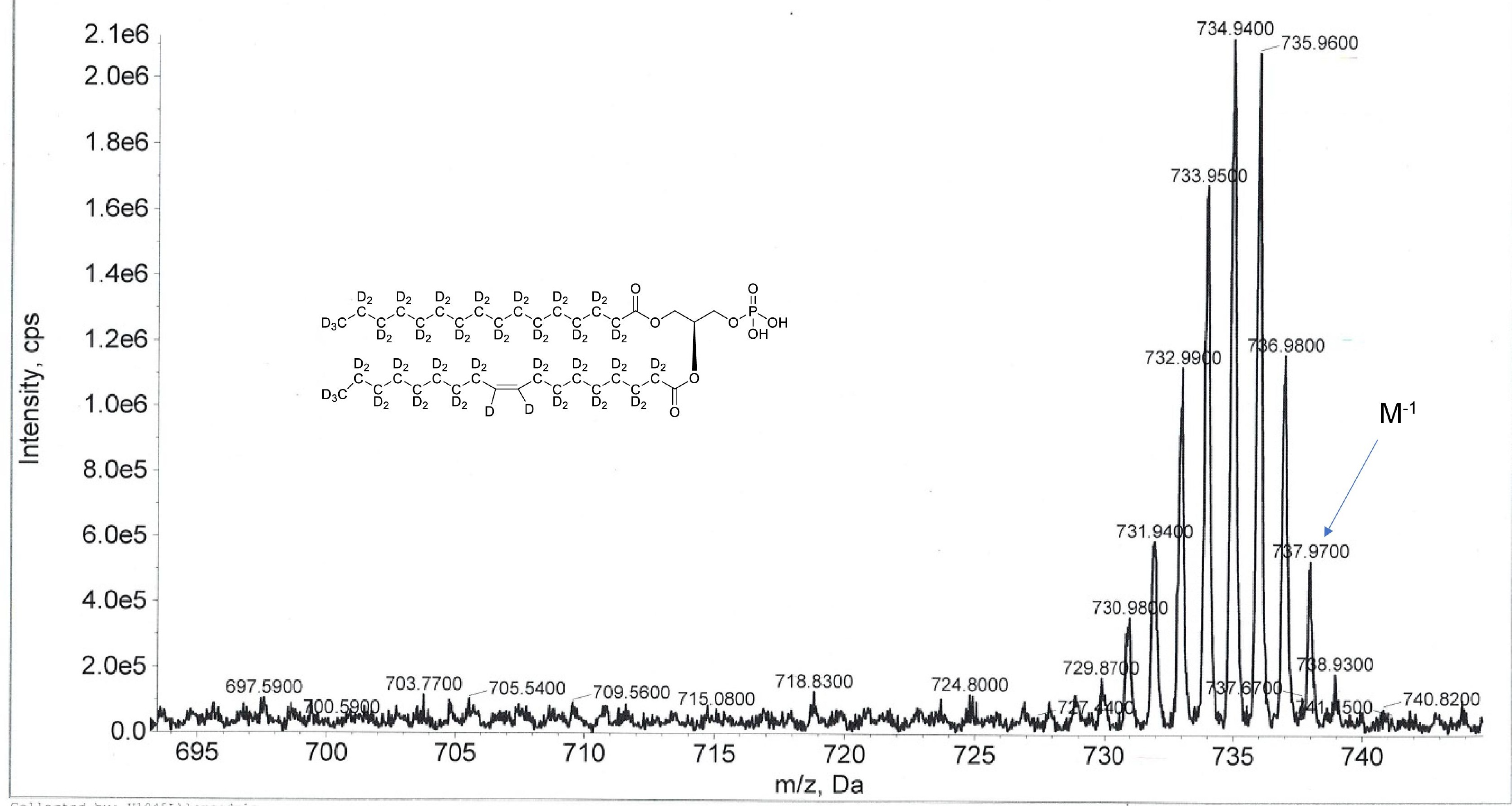




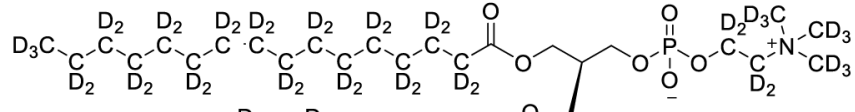

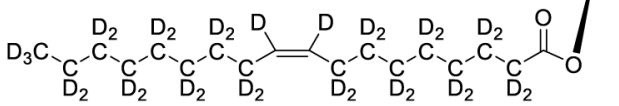




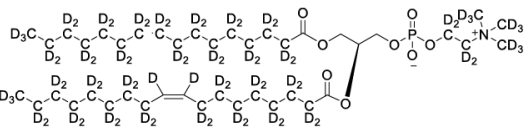

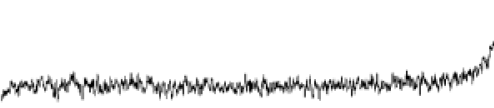

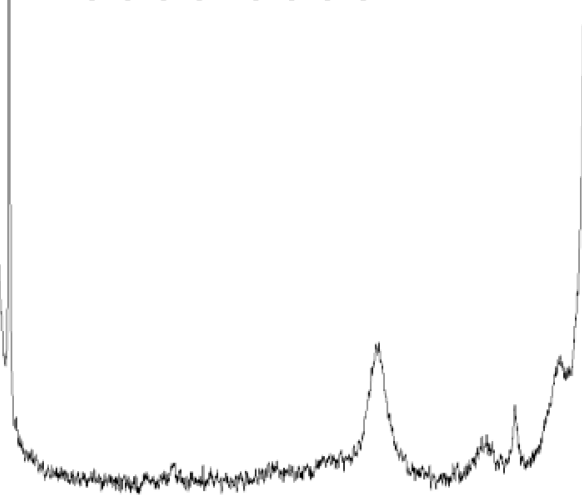

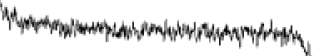




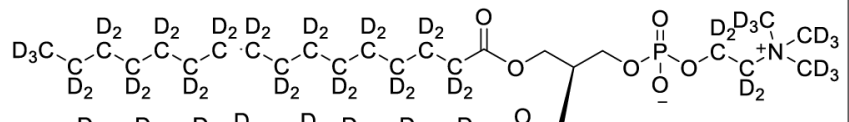

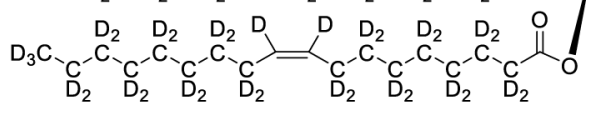




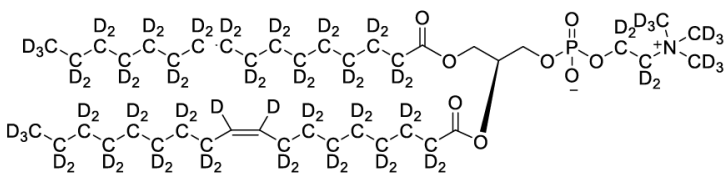




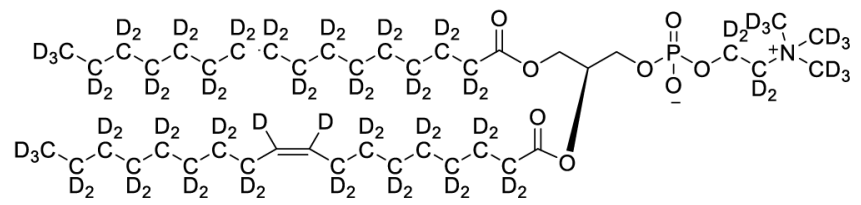

100

$50+0$

$\begin{array}{rrrr}1 & 1 \\ 0 & -50 & -100\end{array}$

$-150$

ppm 


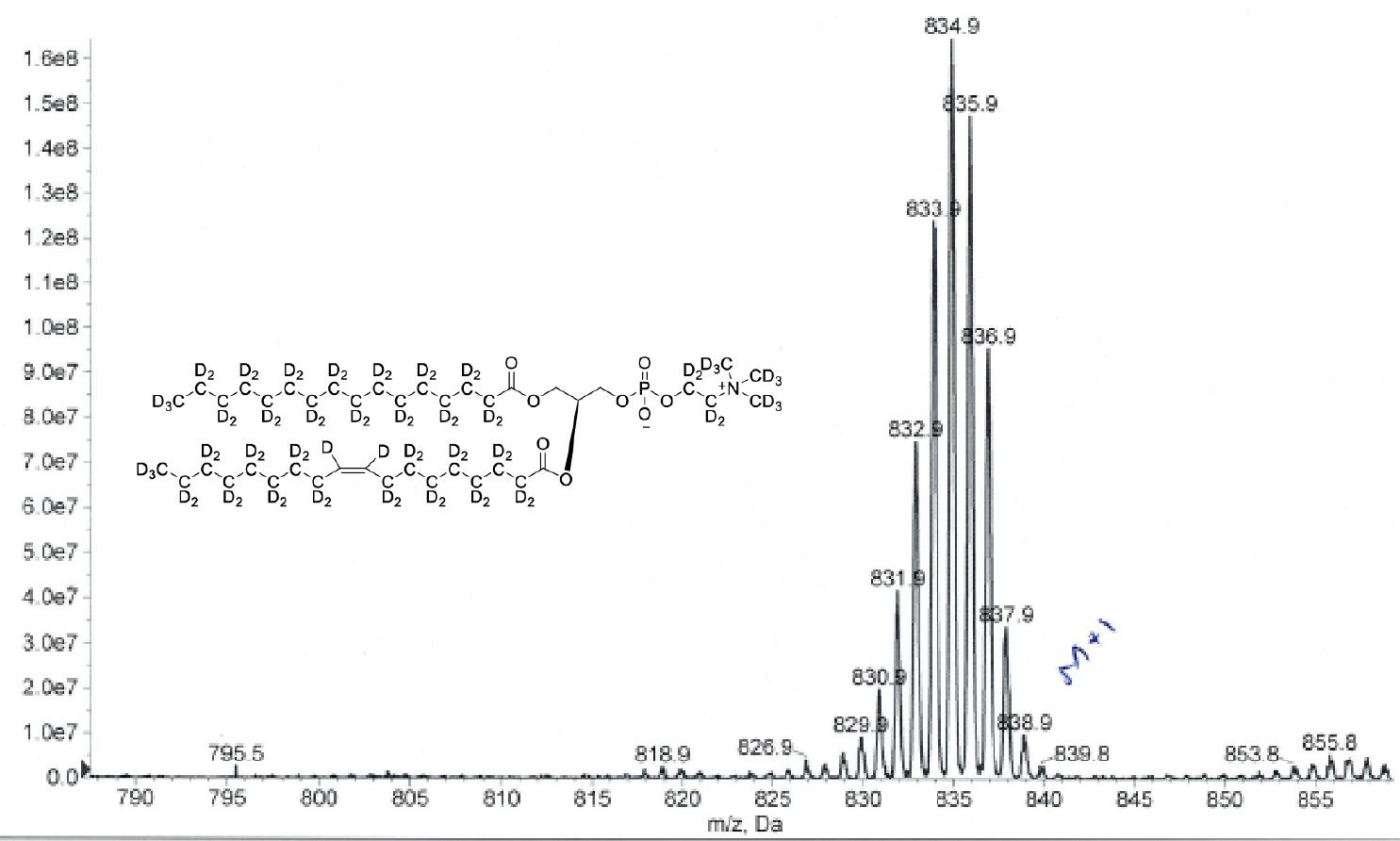




$$
1
$$




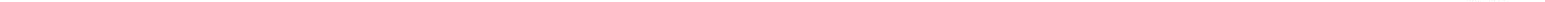




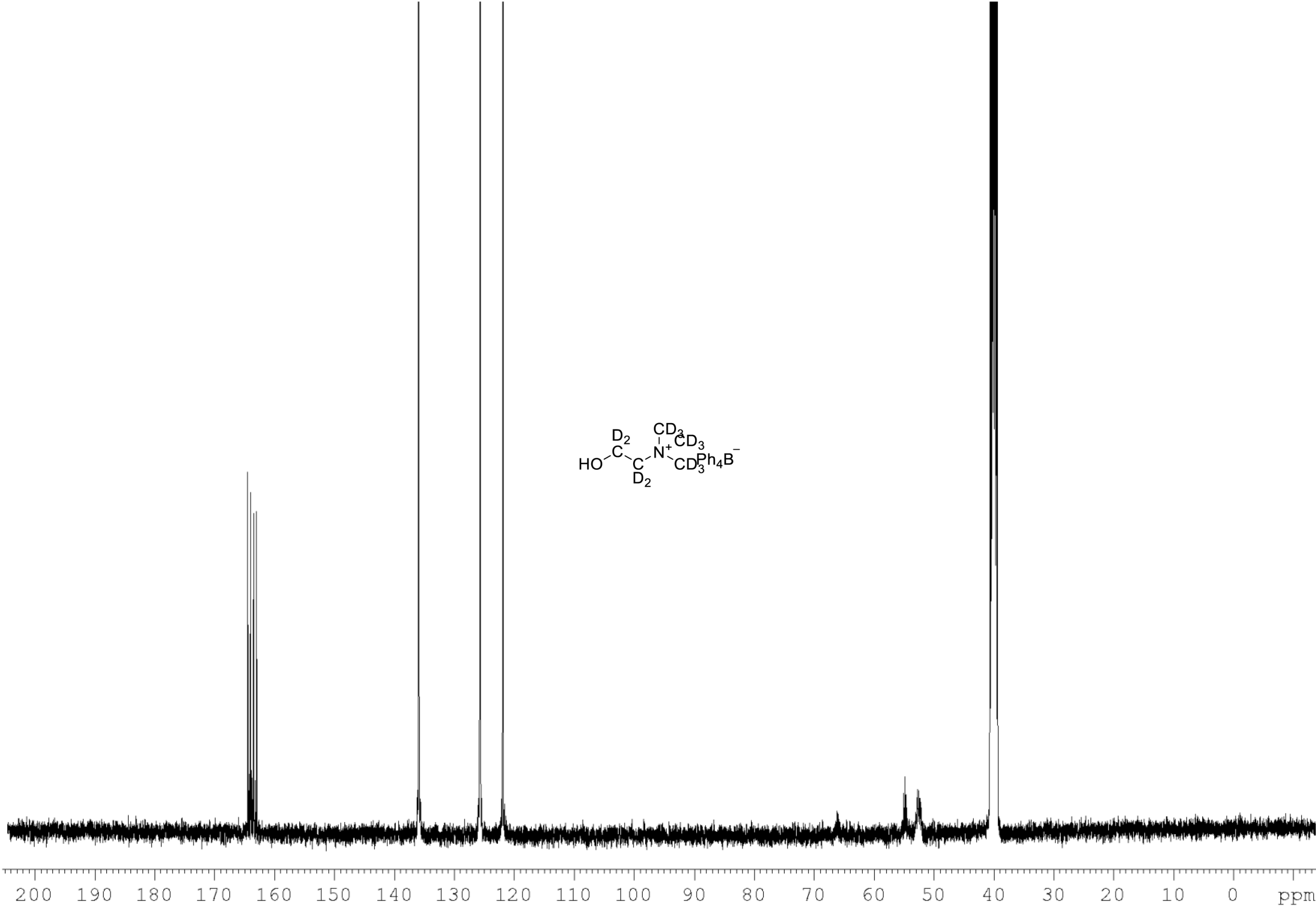




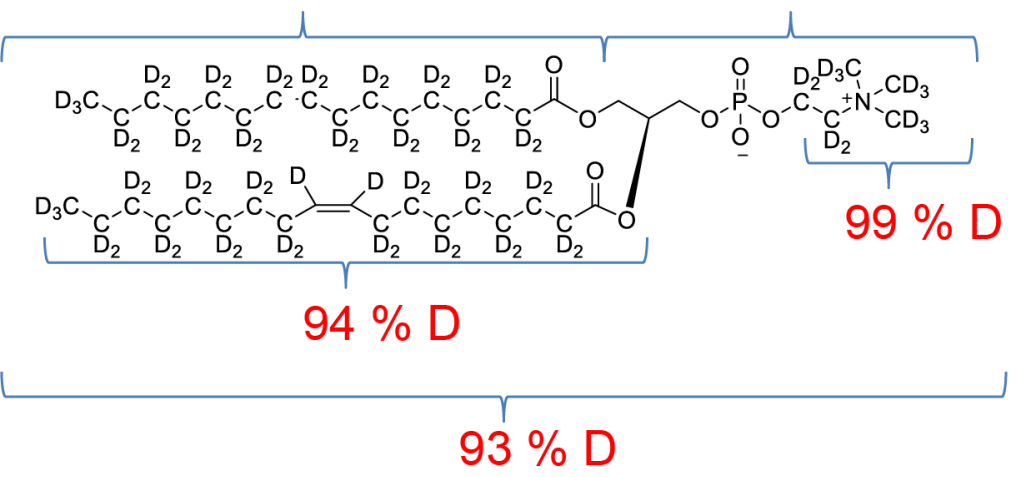

